\title{
Electrochemical Sensors and Biosensors
}

\author{
Danielle W. Kimmel, Gabriel LeBlanc, Mika E. Meschievitz, and David E. Cliffel ${ }^{*}$ \\ Department of Chemistry, Vanderbilt University, 7330 Stevenson Center, VU Station B 351822 , \\ Nashville, TN 37235-1822
}

\begin{abstract}
This review covers advances in electrochemical and biochemical sensor development and usage during 2010 and 2011. In choosing scholarly articles to contribute to this review, special emphasis was placed on work published in the areas of reference electrodes, potentiometric sensors, voltammetric sensors, amperometric sensors, biosensors, immunosensors, and mass sensors. In the past two years there have been a number of important papers, that do not fall into the general subsections contained within the larger sections. Such novel advances are very important for the field of electrochemical sensors as they open up new avenues and methods for future research. Each section above contains a subsection titled "Other Papers of Interest" that includes such articles and describes their importance to the field in general. For example, while most electrochemical techniques for sensing analytes of interest are based on the changes in potential or current, Shan et al. ${ }^{1}$ have developed a completely novel method for performing electrochemical measurements. In their work, they report a method for imaging local electrochemical current using the optical signal of the electrode surface generated from a surface plasmon resonance (SPR). The electrochemical current image is based on the fact that the current density can be easily calculated from the local SPR signal. The authors demonstrated this concept by imaging traces of TNT on a fingerprint on a gold substrate.
\end{abstract}

Full articles and reviews were primarily amassed by searching the SciFinder Scholar and ISI Web of Knowledge. Additional articles were found through alternate databases or by perusing analytical journals for pertinent publications. Due to the reference limitation, only publications written in English were considered for inclusion. Obviously, there have been more published accounts of groundbreaking work with electrochemical and biochemical sensors than those covered here. This review is a small sampling of the available literature and not intended to cover every advance of the past two years. The literature chosen focuses on new trends in materials, techniques, and clinically relevant applications of novel sensors. To ensure proper coverage of these trends, theoretical publications and applications of previously reported sensor development were excluded.

We want to remind our readers that this review is not intended to provide comprehensive coverage of electrochemical sensor development, but rather to provide a glimpse of the available depth of knowledge published in the past two years. This review is meant to focus on novel methods and materials, with a particular focus on the increasing use of graphene sheets for sensor material development. For readers seeking more information on the general principles behind electrochemical sensors and electrochemical methods, we recommend other sources with a broader scope. ${ }^{2,3}$ Electrochemical sensor research is continually providing new insights into a variety of fields and providing a breadth of relevant literature that is worthy of inclusion in this review. Unfortunately, it is impossible to cover each publication and unintentional oversights are inevitable. We sincerely apologize to the

*Fax: (615) 343-1234; d.cliffel@ vanderbilt.edu. 
authors of electrochemical and biochemical sensor publications that were inadvertently overlooked.

\section{REFERENCE ELECTRODES}

Typically, the role of a reference electrode is to remain at a constant potential, thus behaving independently from the properties of the working electrode and those of the solution being measured. ${ }^{2,3}$ This separation between reference electrodes and working electrodes is the basis of accurate electrochemical measurements for a variety of applications. Here, we review recent advances utilizing novel reference electrodes for biologically relevant research, trends in solid-state reference electrode development, and other remarkable reference electrodes that have superior characteristics leading to important implementation in fieldwork and research.

\section{Biological Implications}

Reference electrodes are vital in studying the electrochemical changes of biological systems to both enhance understanding of these complex systems and to aid in the development of treatment for environmental or health-related issues. Recent developments in miniaturization and materials suggest future availability of dependable reference electrodes. $\mathrm{Ag} / \mathrm{AgCl}$ reference electrodes are commonly used in a variety of systems. Unfortunately in some measurements, such as those obtained in biological-based systems, the $\mathrm{Ag} / \mathrm{AgCl}$ electrode promotes inaccuracies and errors that lead to non-reproducible reference potential measurements. Alternatives to traditional $\mathrm{Ag} / \mathrm{AgCl}$ electrodes include $\mathrm{Hg} / \mathrm{HgO}$ reference electrodes, but unfortunately these pose environmental issues. Park et al. ${ }^{4}$ developed a novel silver tetramethylbis(benzimidazolium) diiodide reference electrode that exhibited superior characteristics and reproducibility in acid and alkaline solutions. The promising success of this hybrid electrode provides a suitable alternative to more traditional reference electrodes.

Disposable, miniature reference electrodes are increasingly in demand due to the popularity of microfluidic systems. Zhou et al. ${ }^{5}$ recently reported a miniaturized $\mathrm{Ag} / \mathrm{AgCl}$ reference electrode and its successful application to a microfluidic chip. The electrode exhibited ample stability and adequately sensed heavy metal ions in sea water samples. This novel, miniature reference electrode has potential in a variety of fields where disposability, mass production, and laminar flow are challenges to experiments concerning microfluidic electrochemical measurements. Screening printing has become a useful tool for the fabrication of electrodes. Idegami et al. ${ }^{6}$ were able to develop a disposable sensor containing an $\mathrm{Ag} / \mathrm{AgCl}$ reference electrode, utilizing screen printing technology. They successfully investigated ease of fabrication, stability, and accuracy. Their findings suggest the screen printed $\mathrm{Ag} / \mathrm{AgCl}$ reference electrode has long-term stability and is suitable for mass production, allowing for future biosensing applications.

\section{Solid-State Reference Electrodes}

Reference electrodes have been implemented in a variety of industries, but often times the traditional reference electrode composition is not adequately robust and fails to work properly when subjected to harsh industrial processes. Traditional reference electrodes depend upon a liquid solution for proper potential measurements. Liquid solution-based reference electrodes are in widespread use, however successful miniaturization and mass production is severely limited by continual maintenance as well as contamination control. The alternative is a solid-state reference electrode (SSRE), but typically these devices cannot compete adequately with the reproducibility of liquid-based reference electrodes or be massproduced consistently. Noh et al. ${ }^{7}$ recently developed a novel SSRE using a polyelectrolyte junction. They were able to fabricate a SSRE and a pH-sensing chip that displayed excellent 
reproducibility between synthetic batches, short stabilization time (less than $6 \mathrm{~min}$ ), superior sensing characteristics, and can be readily miniaturized for affordable mass-production.

By using a solid $\mathrm{KCl}$ melt in conjunction with an $\mathrm{Ag} / \mathrm{AgCl}$ component, Vanau et al. ${ }^{8}$ were able to fabricate an SSRE designed specifically for beverage industry applications. They found that this SSRE had a wide range of $\mathrm{pH}$ use, stable potentials, and small drift potentials ( $1 \mathrm{mV}$ at room temperature over three months time). The reported stability and lifetime of this reference electrode make it particularly applicable for the food industry. In addition to food industry, engine diagnostics are utilizing SSRE to assess efficiency. Oxygen sensors have long been utilizing yttria-stabilized zirconia-based potentiometric components. Recently, Mn-based oxides have been studied to find a suitable SSRE to ensure accurate onboard diagnosis for engines. Miura et al. ${ }^{9}$ found $\mathrm{Mn}_{2} \mathrm{O}_{3}$-sensing electrodes to function with excellent sensitivity and has great potential for miniaturization.

Rius-Ruiz et al. ${ }^{10}$ recently reported a carbon nanotube (CNT)-based SSRE. The most successful design tested utilized a photo-polymerised $n$-butyl acrylate polymer in conjuction with SWCNTs, acting as the transducer layer. This type of transducer layer was often superior to alternative solid transducers, and the resulting SSRE proved insensitive to room lighting. The superior characteristics of this reference electrode as well as its ease of fabrication allow for potential widespread usage in a multitude of systems.

\section{Other papers of interest}

To ensure reference electrodes keep up with current miniaturization trends it has become challenging to find suitable materials for all potential applications. One such challenging arena is that of steel corrosion monitoring. Muralidharan et al. ${ }^{11}$ developed a $\mathrm{NiFe}_{2} \mathrm{O}_{4}$ reference electrode due to its superior fabrication cost and ease, and its resistance to corrosion. The resulting electrode exhibited stability and low polarization currents in a calcium hydroxide solution, which is commonly used in concrete environments.

Shibata et al. ${ }^{12}$ examined the stability of an $\mathrm{Ag} / \mathrm{AgCl}$ reference electrode with the novel salt bridge 1-methyl-3-octylimidazolium bis(trifluoromethanesulfonyl)-amide. This bridge was proposed and found to be superior in order to avoid the interference found using alternate ionic liquid salt bridges, such as $\mathrm{KCl}$, while experimenting in phthalate buffer. Further evaluation of stability is necessary, however the initial findings suggest this ionic liquid salt bridge is promising. Huang et al. ${ }^{13}$ recently developed a working system using a Nafion strip membrane as an ion-conducting bridge, allowing for electrochemical measurements in pure water. By using this bridge, it is possible to avoid potential leaching contaminants and gain higher accuracy.

Measuring the electrodeposition of boron melts is a challenge to researchers due to the unknown chemistry of $\mathrm{KF}-\mathrm{KCl}-\mathrm{KBrF}_{4}$. Pal et al. ${ }^{14}$ fabricated an $\mathrm{Ag} / \mathrm{AgCl}$ reference electrode that was found to be reversible, have suitable stability and non-polarizability. This will allow for the first available measurements of $\mathrm{KF}-\mathrm{KCl}-\mathrm{KBrF}_{4}$ chemistry at high temperatures. Zeng et al. ${ }^{15}$ developed a novel reference electrode for use in an alkaline polymer electrolyte membrane fuel cell. Their Pd-coated Pt wire reference electrode studies indicated that water generation and flooding occurs at the anode and can be potentially alleviated by thin alkaline membranes.

There have been numerous enhancements of reference electrode fabrication to allow for suitable stability and size in physiological and environmental systems, allowing for accurate measurements to occur in previously understudied systems. Additionally, there has been an increasing movement toward the development of robust SSREs that can be used in harsh 
industrial processes. The reviewed papers suggest an ongoing trend in specialization of reference electrodes for the further understanding of complex systems.

\section{POTENTIOMETRIC SENSORS}

Potentiometric sensors have been traditionally defined as a zero-current technique that measures the potential across an interface, often a membrane. ${ }^{2}$ The past two years have provided ample advances in the field of potentiometrics and a breadth of reviews have been written for specific niches within potentiometric sensing. One thorough overview of potentiometric sensors was written in 2010 by Bratov et al. ${ }^{16}$ (192 citations). Their review focuses primarily on applications of novel potentiometric sensors and future trends of potentiometric sensor fabrication.

Here we present an assortment of recent publications geared toward the advancement of potentiometric sensors. In the past two years, research has been focused on highlighting the importance of membrane composition using carbon pastes and polyvinyl chloride (PVC) as well as unique ionophores specifically designed for targeted species. Here we review recent advances in ion selectivity, clinical relevance, electronic tongues, and other novel reports on potentiometric sensors.

\section{Ion Selective}

One challenge faced by scientists in fields ranging from medicinal chemistry to environmental toxicity is that of novel ion selective electrodes (ISEs). ISEs typically use an ionophore as the sensing platform to ensure selectivity toward a specific ion of interest. The continual rise of heavy metal and ion usage in industrial processes makes ion selective sensors vital for the proper sensing and quantification of potential pollutants. In the past two years there have been a plethora of ISE advances with cations, anions, and neutral species. These advances have enabled researchers to measure minute amounts of ionic species, which can eventually aid in pollution containment and pharmaceutical screening.

Cation-Carbon pastes are becoming increasingly useful in membrane composition to enhance the sensing capability of the electrode. Faridbod et al. ${ }^{17}$ recently developed an ISE for rapid and sensitive determination of $\mathrm{Yb}^{3+}$ ions. The potentiometric sensor was developed based on carbon paste and multi-walled carbon nanotube (MWCNT) membranes. N'-(1oxoacenaphthylen-2(1H)-ylidene) furan-2-carbohydrazide proved to have great selectivity for $\mathrm{Yb}^{3+}$ ions and allowed for better potentiometric responses than typical carbon paste electrodes (CPEs). The use of room temperature ionic liquids (RTIL), instead of the typically used paraffin, contributed greatly to the sensor performance. Another use for carbon paste electrodes based on MWCNT, RTIL, and nano-silica was devised to fabricate an $\mathrm{Er}^{3+}$ potentiometric sensor. $\mathrm{N}^{\prime}$-(2-hydroxy-1,2- diphenylethylidene) benzohydrazide was used due to its strong interaction with $\mathrm{Er}^{3+}$ ions. ${ }^{18}$ These sensors improved all operational metrics such as the limit of detection and sensitivity, making these potentiometric sensor responses better than currently used sensors. A second novel CPE fabricated by Faridbod et al. ${ }^{19}$ provides an alternative device for $\mathrm{Ho}^{3+}$ sensing. The sensor composition is made up of paraffin oil, graphite powder, MWCNTs, and N-(1-thia-2-ylmethylene)-1,3benzothiazole-2-amine as the ionophore. The findings suggested a Nernstian slope over a wide concentration range in addition to selectivity, rapid response time, stability, and ease of preparation.

Copper is a necessary atom in biological processes, however in high concentrations copper can displace metal ions in vivo, leading to illness or fatality. Unfortunately, many biochemical processes carried out in industrial settings depend on the presence of copper ions, necessitating selective sensors to reduce the potential for copper toxicity. Current 
techniques to quantify residual copper in a given sample are time consuming and expensive. A recent report by Ganjali et al. ${ }^{20}$ has shown that 7-hydroxy-3-(2-methyl-2,3dihydrobenzo[d]thiazol-2-yl)-2H-chromen-2-one has great selectivity toward $\mathrm{Cu}^{2+}$ ions. The authors in this study developed a CPE utilizing the selective behavior of 7-hydroxy-3-(2methyl-2,3-dihydrobenzo[d]thiazol-2-yl)-2H-chromen-2-one towards $\mathrm{Cu}^{2+}$. Modification of the CPE was done using MWCNTs and nanosilica and was successful in measuring the target ion in water samples. They report a Nernstiain slope, a working $\mathrm{pH}$ range of 3-6.0, and a relatively short response time (13 s). Another novel copper selective electrode was fabricated by Mashhadizadeh et al. ${ }^{21}$ using a macrocyclic ligand, such as crown ethers with oxygen atoms substituted with nitrogen and sulfur atoms, to potentiometrically measure copper abundance in samples. This affords selectivity to $\mathrm{Cu}^{2+}$ ions over other ions and allows for good operating characteristics. Additionally, the use of macrocyclic ligands provides an easy fabrication method and is cost effective for sample analysis. Kopylovich et al. ${ }^{22}$ recently reported the use of 1-Phenyl-2-(2-hydroxyphenylhydrazo)butane-1,3-dione as an ionophore, which allowed for good selectivity for copper when compared with alkali metals. The resulting sensor allowed for a Nernstian response throughout a wide concentration range and excellent characteristics compared with alternative copper sensing electrodes. Petković et al. ${ }^{23}$ presented a novel $\mathrm{Cu}^{2+}$ ISE using $\mathrm{N}, \mathrm{N}^{\prime}, \mathrm{N}^{\prime \prime}, \mathrm{N}^{\prime \prime \prime}-$ Tetrakis(2pyridylmethyl)-1,4,8,11-tetraazacyclotetradecane as the ionophore in a PVC matrix. The novel ISE construction is simple, allowing for a cost effective, rapid measurement of copper in a mixed solution with ethylenediaminetetraacetic acid (EDTA). The resulting measurements were comparable with commercial sensors with a superior working $\mathrm{pH}$ range as well as a longer lifetime, suggesting a practical use for these novel copper sensors.

Proper composition of ISE membranes can enhance the response time for ionic sensing. PVC has proven to be suitable for ISE construction allowing for ease of construction, suggesting a potential widespread usage of novel electrodes. In the past two years, PVC containing ISEs have performed in a variety of industrial studies including medical research and water contamination experiments, showing the versatility of these electrodes for potential applications in field work. Hosseini et al. ${ }^{24}$ used a PVC membrane electrode using $\mathrm{N}, \mathrm{N}^{\prime}$-phenylenebis (salicylideaminato) for selective $\mathrm{Zn}^{2+}$ sensing. They reported successful sensing over a wide concentration range, independent of $\mathrm{pH}$, low detection limit, and short response time. Another biologically important ion is the rare earth element terbium, as its therapeutic application has become increasingly studied in recent years. Hassan et al. ${ }^{25}$ developed a novel potentiometric electrode using the Schiff base, N,N'-bis(5nitrosalicylidene)-2-aminobenzylamin as a selective $\mathrm{Tb}^{3+}$ ionophore. Their results indicate that a mixture of membrane components (sodium tetraphenyl borate, nitrobenzene, PVC) allow for a minimal response time of $10 \mathrm{~s}$ and successful sensing independent of $\mathrm{pH}$. Recent evidence suggests that aluminum could contribute to the generation of Parkinson's disease and Alzheimer's disease, as well as being known to interfere with phosphorus metabolism. While trace amounts of aluminum are vital, overexposure due to continual use in industry, construction, and useful daily products makes selective aluminum sensors vital. Ma et al. ${ }^{26}$ developed an N,N'-propanediamide bis(2-salicylideneimine) sensing carrier in a PVC matrix membrane to allow for selective $\mathrm{Al}^{3+}$ ion sensing. This sensor afforded superior working characterizations compared with current sensing techniques and allows for easy construction, lending itself readily to $\mathrm{Al}^{3+}$ determination in real samples.

Residual iron in aquatic systems can greatly impact the environment and lead to toxicological issues. To measure the concentration of iron, current techniques used are expensive and require trained analytical chemists. Recently, a potentiometric device was made by Motlagh et al. ${ }^{27}$ to speed up water sample analysis in a cost efficient and accessible way. Here, they used a PVC membrane electrode and a coated graphite electrode with 1phenyl-3-pyridin-2-yl-thiourea (PPT) as the iron carrier. The most successful composition 
tested was PVC/DBP/NaTPB/PPT (30:64:2:5, w/w; mg). This sensor provided an ironselective membrane that allowed usage of the potentiometric sensor at a wide temperature range, independent of $\mathrm{pH}$, and a rapid response time $(<10 \mathrm{~s})$. Sensing of lead ions has also become important, as researchers have investigated its toxic effects to both health and the environment. Abbaspour et al. ${ }^{28}$ constructed an electrode with a phenyl hydrazone derivative coating on platinum wire. The sensor membrane was made of PVC and enabled Nernstian slope measurements over a wide concentration range. The sensor also provided great working characteristics and exhibited selectivity for lead over potential interfering ions. This sensor enables rapid detection of lead contamination and can be practically used in a variety of fields. Ganjali et al. ${ }^{29}$ also enhanced $\mathrm{Pb}^{2+}$ ion sensing by using a MWCNT and nanosilica membrane. Their unique composition of the electrode provided a Nernstian slope over a wide linear range, stability, and excellent characteristics.

Anion-Other important advances with potentiometrics have provided novel technologies that will enhance research capabilities and quality of life. One such potentiometric sensor niche is that of selectively sensing anions. Anion selectivity sensing poses problems for ISEs, due to the availability of ionophores having discriminating interaction with the target. Traditionally, macromolecules with strong central metal atoms have been utilized for cation detection, however anion selectivity remains challenging.

PVC membranes containing ISEs, described earlier, have also proven useful in anion selective electrodes. Chloride ions are of great importance for all biological systems, as well as many industrial processes. Due to the growing need for miniaturization with chloride sensing, Álvarez-Romero et al.$^{30}$ successfully developed a chloride ion selective, composite electrode. They were able to accomplish this by using a graphite-based composite containing epoxy resin, doped with chloride ion-doped polypyrrole. The comparison of this potentiometric sensor with other recently published sensors revealed equivalence in analytical parameters, superior selectivity, and shows potential for future miniaturization. Zahran et al. ${ }^{31}$ proposed a novel class of halide receptors, triazolophanes, which allow for selectivity toward chloride and bromine. The size and organization of the macrocyclic scaffold on the PVC-based sensor was found to play an important part in selectivity and high binding constants. Fluoride selective sensors allow for rapid detection of toxic organic fluorophosphates, which can contaminate drinking water. Kang et al. developed and characterized a $\mathrm{Sc}^{3+}$ octaethylporphyrin based fluoride selective electrode. The only interference with selectivity was salicylate, however the sensor allows for a binding constant for fluoride of $\beta_{\mathrm{Sc}(\mathrm{III}) \mathrm{OEP}-\mathrm{F}}=10^{12.8}$ and a lower detection limit. ${ }^{32}$

Neutral Species-Sensing uncharged molecules has been a challenge for researchers due to traditional uses of ionophores that sense anionic or cationic species. The use of molecularly imprinted polymers (MIPs) has been greatly increasing in the search to enhance ISEs. Until recently, ISEs have been unable to adequately measure uncharged molecules. Liang et al. ${ }^{33}$ developed a novel strategy using a polymeric membrane ISE to detect neutral molecules. Their ISE utilizes an MIP as a sensing element and an indicator ion that is similar in structure to the targeted neutral species. The neutral species studied was that of chlorpyrifos, an organophosphate pesticide. The potentiometric sensor incorporates two steps: accumulation of neutral species in the membrane phase and removal of the indicator ion and potential measurement with the ISE. This novel sensing device reported by the authors is selective, sensitive, and has a lower detection limit for chlorpyrifos sensing.

Detection of hydrocarbons as waste products in industrial systems has become increasingly important for environmental protection in the past few years. Single-walled carbon nanotubes (SWCNTs) have been shown to have an adsorption affinity for these potential pollutants, primarily due to their $\pi-\pi$ and hydrophobic interactions, suggesting SWCNT use 
in potentiometric sensing devices. Washe et al. ${ }^{26}$ found that their sensor was capable of detecting these uncharged species through the nanotube-solution interface that allowed for a double layer capacitance change. This promising device has good working characteristics, is cheaper and easier to use than traditional methods, and could be miniaturized for future industrial sensing. $\mathrm{CO}$ sensing has also proved vital to environmental toxicity measurements. Park et al. ${ }^{34}$ recently developed a miniature potentiometric titania-based sensor for the purpose of carbon monoxide detection. To enhance sensitivity, a novel mixture of n-type and p-type electrodes were fabricated on opposite sides, yielding a sensing resolution of $10 \mathrm{ppm} \mathrm{CO}$ concentration variations.

\section{Water Monitoring}

Water pollutants from industrial and agricultural processes can be hazardous to aquatic and agrarian life. Recent developments in water monitoring sensors enable detection of pollutants as well as assessment of water quality. Surface water pollution has been amplified in recent years due to increasing surfactant production. Unfortunately, the typical method of surfactant detection is a time consuming two-phase process requiring restricted, toxic chemicals. Madunić-Čačić et al. ${ }^{35}$ present a novel potentiometric sensor based on a PVCplasticized liquid membrane housing hexadecyltrioctadecylammonium-tetraphenylborate as the electroactive ion-pair. Their findings included a Nernstian response, a relatively rapid response time, and an agreement with the standard two-phase procedure. This sensor suggests future use as a cost effective and rapid replacement for the current two-phase system. Quantifying dissolved oxygen in water samples is a vital measurement to assess the quality of water, which greatly impacts the environment and health. A new potentiometric sensor based on dissolved oxygen sensing, was proposed and tested by Zhuiykov et al. ${ }^{36}$ using a $\mathrm{Cu}_{2} \mathrm{O}$-doped $\mathrm{RuO}_{2}$ electrode. This doping strategy allowed for less fouling and enhanced sensing properties. These studies imply that a low concentration doping of the $\mathrm{RuO}_{2}$ electrode improves sensitivity, selectivity, and does not change the ruthenium chemical state. Tetracycline is a clinically relevant antibiotic, however due to its frequent use there is an increasing environmental and biological concern as its infiltration of water systems increases. Cunha et al. ${ }^{37}$ utilized $\beta$-cyclodextrin as the ionophore in their ionselective potentiometric sensor for tetracycline. These studies indicated superior working characteristics of this ion-selective electrode when compared with current tetracycline sensors, and suggest a future use for pharmaceutical screening.

\section{Clinically Relevant}

Potentiometric sensors have become increasingly useful in biomedical research.

Pharmaceutical development, disease screening, and disease research are just a few areas currently using sensors to enhance knowledge and treatment options. PVC has played an important role in membrane fabrication for clinically relevant sensors as well as ISEs and their versatility can be seen by the variety of species sensed.

Domperidone is a clinically useful pharmaceutical for a wide variety of stomach ailments. Unfortunately, typical domperidone concentration levels are measured with expensive analytical techniques. Kumar et al. ${ }^{38}$ were able to fabricate a sensor capable of selective determination of domperidone. This was accomplished by two separate sensors, made of a PVC membrane and a carbon plate, with the electroactive ion pair domperidonephosphotungstic. Both cost-effective sensors were characterized through analysis of linear range, detection limit, and response time. The results indicated that the carbon-based sensor was superior in these characterizations and remained highly selective over other ions.

Abounassif et al. ${ }^{39}$ describe the fabrication and testing of a potentiometric sensor using PVC membranes with three different electroactive materials to sense arecoline, which is thought 
to promote cancerous growth. The materials, AR-tetraphenyl borate, AR-

phosphomolybdate, and AR-phosphotungstate, proved successful in accurately and precisely measuring arecoline in pure solutions and in human saliva. The comparative success of these sensors to traditional methods makes this a superior system due to its feasibility, low cost, and limited sample preparation.

Numerous active materials for use in polymeric membranes have been studied in the past two years. One promising material is that of $\mathrm{Mn}^{3+}$ porphyrins due to its selectivity and reversibility in binding. Vlascici et al ${ }^{40}$ evaluated the use of manganese porphyrins in PVC and sol-gel membranes versus other available diclofenac sensors and found that their Mnporphyrin sensor had the best sensitivity. The sensor had good working characterizations and displayed comparable results to traditional HPLC methods in pharmaceutical preparation determinations of diclofenac in drugs. Using MIPs, a new PVC-based potentiometric sensor was fabricated to sense promethazine. Alizadeh et al ${ }^{41}$ found that the success of sensor performance was based upon the monomer composition of any MIP formed. The sensor was tested against samples of blood and urine and produced reliable results, suggesting the future use and importance of MIP-based potentiometric sensors in a clinical setting.

In pharmaceutical research, quantification of active ingredients is vital to drug formulation. Current analytical methods are time consuming and costly, making potentiometric sensors a viable and attractive alternative. A new potentiometric device recently targeted one clinically relevant compound, terazosin hydrochloride. Ganjali et al. ${ }^{42}$ first utilized computational chemistry to investigate terazosin interaction with various ion-pairs. The sensor membrane incorporated terazosin-tetraphenyl borate ions and plasticizers. This fabrication proved to have excellent working parameters compared with alternate sensors, including a lower detection limit $7.9 \times 10^{-6} \mathrm{M}$, a pH range of $3.2-5.5$, and a fast response time $(\sim 15 \mathrm{~s})$.

The nervous system relies heavily on cholinesterases (ChEs) for proper function in catalyzing the hydrolysis of acetylcholine into choline. The current measurements of ChEs are extremely sensitive, but cannot quantitatively assess ChEs in situ. Additional problems with the current methods are their high cost and involvement of trained personnel. Here, Khaled et al. ${ }^{43}$ provided a new methodology for the production of low cost ChEs sensors that retain the optimal characteristics for ChEs sensing. Their sensor employed a screenprinted carbon material, which allows for mass production, cost efficiency, and a longer shelf life (6 months) per electrode. Their usage of a novel potentiometric butyrylcholine proved accurate in measuring ChEs from blood samples, making this a novel tool for inexpensive clinical assays. Microscopy and multi-welled plates are traditionally used for cytotoxicology examination in vitro. Unfortunately, these two techniques are laborious and require expensive instrumentation and can be time consuming. Building off of the unique electrochemical properties of the cell, Wang et al. ${ }^{44}$ decided to utilize potentiometric sensors to quantify the impact of environmental toxins in a non-invasive, rapid, and continuous way. They successfully measured potential changes of a cellular membrane caused by exposure to toxic chemicals. The various electrode substrates allowed for numerous toxic chemicals to be studied on their polymer based biosensor. Controlling the type and amount of doping allowed for kinetic information to be gathered in real-time, throughout the course of the experiment.

\section{Electronic Tongues}

Electronic tongues (E-tongues) have become increasingly studied due to their industrial capabilities to ensure safety and health. In 2010, Riul et al. ${ }^{45}$ published a minireview on current E-tongue advances and technology (212 citations). Another 2010 review by Valle ${ }^{46}$ 
focuses on the electrochemical sensors used for E-tongues (138 citations). Here we present a few new advances in E-tongue development and application, but also other novel potentiometric sensors.

Unknown gluten content in foodstuffs can lead to serious complications for those suffering from celiac disease. There is no current technique that is readily available for rapid, portable analysis of gluten content, and the misuse of labeling has caused widespread concern. Recently, Peres et al. ${ }^{47}$ developed a solid-state potentiometric non-specific sensor to function as an E-tongue device for the sensing of gluten in food samples. The samples were subjected to ethanol extraction and centrifugation prior to measurement, making sample preparation easy and cost effective. They found that their E-tongue device had good sensitivity (1-2 mg/kg) and could reproducibly differentiate between "Gluten-free" and "Gluten-containing" food samples. In an effort to standardize food and beverage profiling by an analytical technique, Hruškar et al. ${ }^{48}$ proposed utilizing a novel E-tongue to qualitatively assess probiotic fermented milk. They were able to successfully compare a sensory analysis with their E-tongue and found a high correlation with the results, indicating that the Etongue could enhance the speed, cost, and accessibility of sensory analysis in the future.

Another interesting and practical use for E-tongues is analysis of beer bitterness. Typically, bitterness is assessed with the European Bitter Units method, which is time consuming, costly, and contaminates the beer samples with organic solvents. Newer technologies allow for chromatographic methods to assess beer bitterness, but these are also time consuming and rely on expensive instruments and skilled technicians. Polshin et al ${ }^{49}$ devised a potentiometric E-tongue designed to examine numerous physiochemical parameters of beer, including its bitterness. The potentiometric sensor designed at St. Petersburg University gave promising results in predicting the physiochemistry of the beer samples and shows promise for future use in the rapid analysis of beer.

Meat spoilage is assessed based upon sensory and biochemical tests, both requiring highly trained individuals, expensive techniques, and time consuming processes. Gil et al. ${ }^{50}$ have presented new data on the use of potentiometric devices made from $\mathrm{Au}, \mathrm{Ag}, \mathrm{Cu}, \mathrm{Pb}$, or $\mathrm{Zn}$ for use in E-tongue sensing. They were able to show a correlation between the obtained response and degrading indicators such as $\mathrm{pH}$, microbes, and nucleosides. Their methodology is rapid and inexpensive allowing for future application to an assortment of fields.

\section{Other Papers of Interest}

Quantification or characterization of compounds in minute quantities is becoming more and more necessary for biological and environmental studies. Potentiometric sensors based on ion-sensitive field-effect transistors have been useful tools to study the changes in the dielectric-electrolyte interface potential; however studying organic transistors using ionsensitive field-effect transistors has proven difficult due to reproducibility and stability issues, as well as Faradaic leaking. Spijkman et al. ${ }^{51}$ report that the changes in threshold voltage can be measured successfully and sensitivity can be enhanced by proper ratio in each gate of the dual-gate transducer.

$\alpha$-Amylase is an enzyme in many biological systems and is responsible for the breakdown of some glycosidic linkages in starch, providing accessible sugars for energy. Multiple industries including clinical diagnostics and food production rely $\alpha$-amylase sensing, which is performed by four assay types. These assays are mainly spectrophotometric, and require tedious sample preparation and are often high in cost. Sakač et al. ${ }^{52}$ present a theoretical approach to measuring $\alpha$-amylase using a potentiometric sensor. The sensor design employed a platinum redox electrode and would be able to measure triiodide released from 
$\alpha$-amylase promoted starch degradation. The sensor is proposed to be easily and inexpensively fabricated into a miniature, portable system or inserted into a microfluidic system.

Within just two years there has been a breadth of novel potentiometric sensors and applications in a variety of fields. Many sensors have employed carbon pastes or PVC mixtures in addition to innovative ionophores to ensure membrane composition that enables superior sensing of select species. Over the past two years we have seen this trend of perfecting target-specific sensor composition grow immensely and become applicable and useful in biomedical, industrial, and environmental research. This compilation of research suggests that target-specific potentiometric sensors will become increasingly vital and in widespread use in the near future.

\section{VOLTAMMETRIC SENSORS}

Voltammetry provides an electroanalytical method for deriving information about one or more analytes by measuring the current as a function of the potential. Several types of experiments may be performed to gather information from voltammetry including cyclic voltammetry, squarewave voltammetry, and stripping voltammetry to name a few common techniques. Recent review articles have focused on specific applications or methods for voltammetric sensors. The recent review by Gupta et al ${ }^{53}$ focuses on the various application of voltammetry to pharmaceutical analysis from 2001-2010 (279 citations). Dogan-Topal et al. ${ }^{54}$ have also published a review focused on pharmaceutical analysis, however their review has an additional focus on square wave voltammetry (218 citations). Besides listing the various advances in the field from 1997-2010, the authors describe the underlying theory and practical information for performing square wave voltammetric analysis. Alghamdi ${ }^{55}$ has published a recent review dealing with the application of stripping voltammetry in the analysis of food (132 citations). Bustos and Godinez ${ }^{56}$ have published a review on the use of Prussian Blue-dendrimer nano-composits as a useful material for electrochemical sensors (217 citations). Finally, a recent review by Jacobs et al. ${ }^{57}$ discussed the recent literature on the use of CNTs in electrochemical sensors for biomolecules such as neurotransmitters, proteins, and DNA (202 citations). The reviewers conclude that future direction for this field will focus on sensor miniaturization, isolation of specific nanotube allotropes, and the use of different varieties of CNTs to exploit their various advantages synergistically.

\section{Carbon based materials}

Carbon based electrodes have been widely used in voltammetric studies for a variety of reasons, including low cost, availability, stability, and the ability to easily modify the morphology of carbon. There are a number of carbon-based electrodes including glassy carbon (GC), polycrystalline boron doped diamond (pBDD), carbon nanotubes (CNTs), and most recently graphene. In order to understand the potential improvements of newer carbon materials, Guell et al. ${ }^{58}$ investigated the characteristics of three distinct carbon-based electrodes: GC, pBDD, and CNTs. Through the detection of the neurotransmitter serotonin, they found that "pristine" CNT networks exhibited background current densities that were two orders of magnitude lower than GC and twenty times lower than pBDD. Furthermore, they found that pBDD underwent electrode fouling to a lesser extent than did CNTs, and that this fouling could be further reduced via careful selection of the potential range. Apetrei et al. ${ }^{59}$ studied the sensing properties of CPEs prepared using graphite, carbon microspheres, or MWCNTs. They found that CPEs prepared with MWCNTs had the lowest background current, while electrodes prepared with graphite had the lowest detection limit for antioxidants. Electrodes prepared with carbon microspheres showed the best performances in terms of kinetics and stability. Finally, they demonstrated how an array of the three types 
of electrodes could be used to discriminate between various antioxidants based on their chemical structure and reactivity.

The use of carbon as an electrode tip has numerous advantages including mechanical strength and inhibition of water electrolysis. Prior to the work of Sripirom et al. ${ }^{60}$ however, methods for producing carbon ultramicroelectrodes (UMEs) required complex and expensive instrumentation. Utilizing electrochemical etching, they demonstrated a simple technique for producing conical UMEs on the order of $1 \mu \mathrm{m}$. The ability to efficiently prepare UMEs will enable their use in more electrochemical sensing studies in the future.

Graphene, a relatively new two-dimensional material, has been the focus of much research in the field of electrochemical sensors due to its unique properties. A majority of recent electrochemical studies involving graphene have been performed using reduced graphene oxide. In order to gain a greater understanding of the intrinsic electrochemical characteristics of graphene, Lim et al. ${ }^{61}$ have performed systematic studies of crystalline epitaxial graphene prepared on silicon carbide. They found that pristine graphene had slow electron transfer kinetics when compared to anodized epitaxial graphene. This effect was attributed to the increase in edge plane defects, thus the higher the defect density the greater the electron transfer kinetics. The anodized epitaxial graphene was then shown to be a superior electroanalytical platform for a wide range of biomolecules compared to other carbon based electrodes, with the ability to resolve the anodic peaks of all four nucleic acids in both single and double stranded nucleic acids. The importance of accessible graphene sheet edges is further demonstrated by Ambrosi and Pumera ${ }^{62}$ who demonstrated that stacked graphene nanofibers have superior electrochemical performance for the oxidation of DNA bases than CNTs, edge plane pyrolytic graphite, graphite microparticles, and GC. The difference in the number of edge sites can be seen in Figure 1. Chang et al. ${ }^{63}$ were able to control the density of oxygen-containing functional groups on nanoplatelets of graphitic oxide by varying the temperature of microwave-assisted hydrothermal elimination. They determined that the edge-plane-like sites of the electrode were the electroactive sites, which were demonstrated to be useful for increasing the current response and peak shift between uric acid and ascorbic acid. This effect was attributed to ability for these molecules to form hydrogen bonds with the graphitic oxide. Wang et al. ${ }^{64}$ demonstrated how reduced graphene oxide sheets could be prepared to modify a GC electrode in order to analyze the oxidation of hydrazine via cyclic voltammetry. Their simple vacuum deposition of reduced graphene oxide sheets to modify a GC electrode is of particular interest for future studies. Further fundamental understanding behind the electrochemistry of graphene and its derivatives or composites will enable the optimization of this new material for various electrochemical sensing methods.

Graphene has recently been incorporated in nanocomposites in order to couple its unique properties with the properties of other nano-materials. Yue et al ${ }^{65}$ have developed a sensing platform composed of single-layer graphene nanoplatelet and heme protein. They found that single-layer graphene nanoplatelet provided a biocompatible microenvironment for protein immobilization with suitable electron transfer characteristics. The resulting composite film was characterized and used for the indirect determination of nitrite. Using a polyol process, Zhang et al. ${ }^{66}$ prepared a $\mathrm{Cu}_{2} \mathrm{O}$-graphene nanocomposite, which was then deposited on a GC electrode. The modified electrode was then used to selectively determine the concentration of dopamine with a detection limit of $10 \mathrm{nM}$. A $\mathrm{TiO}_{2}$-graphene nanocomposite was prepared by Fan et al. ${ }^{67}$ using hydrolysis and in situ hydrothermal treatment. The composite was then used to modify a GC electrode, which showed significant improvement in the electrocatalytic activity towards adenine and guanine. Du et al. ${ }^{68}$ have prepared a $\mathrm{ZrO}_{2}$-graphene nanocomposite using an electrochemical deposition method. Due to the strong affinity of the composite for phosphoric moieties, it can be used as capturing agent or as a sensing material for organophosphorous agents. 
Other carbon-based materials have also been used in nanocomposites in order to couple their unique features with the selectivity and catalytic ability of other materials. Nanocomposites based electrodes have shown improved selectivity by inhibiting the interference reaction at the electrode. Komathi et al ${ }^{69}$ have demonstrated nanomolar detection of dopamine through the use of a new nanocomposite made up of MWCNTs, a grafted silica network, and gold nanoparticles. They attribute this increased sensitivity to the discriminate sites for the analyte of interest and interfering molecules. Yang et al. ${ }^{70}$ have prepared gold nanoparticles which were then tethered to an ethylenediamine/MWCNT modified GC electrode. The high surface area from the nanotubes combined with the increased catalytic ability of the gold nanoparticles allowed for the selective determination of rutin in the presence of ascorbic acid. Gong et al. ${ }^{71}$ have utilized a nanofiber matrix decorated with gold and platinum nanoparticles to modify a GC electrode. The porous structure of the resulting electrode provides a large effective surface area that behaves like a microelectrode ensemble for the detection of low concentrations of $\mathrm{Hg}^{2+}$. Huo et al. ${ }^{72}$ have developed a method for modifying a gold electrode with carbon nanofibers followed by the electrodeposition of gold nanoparticles. The composite modified electrode was found to have excellent sensitivity and selectivity properties, which enabled the simultaneous determination of catechol and hydroquinone. They also note that the electrochemical properties and inherent biocompatibility make this composite suitable for use in amperometric biosensors. Liu et al. ${ }^{73}$ have developed a novel electrode based on a $\mathrm{PbO}_{2}$-MWCNT-RTIL composite. The use of this composite to modify a GC electrode was found to not only amplify the oxidation peaks for adenine and guanine, but also decreased the oxidation peak potential significantly.

Carbon materials have also been incorporated into active and inert matrices. As an active matrix, ionic liquids possess unique properties such as wide electrochemical windows and high ionic conductivity. Guo et al. ${ }^{74}$ have developed an ionic liquid modified graphene composite in order to couple the surface-to-volume ratio and conductivity of graphene with the ability for ionic liquids to disperse graphene. The resulting ionic liquid-graphene paste electrode proved to be superior to ionic liquid-CNT and ionic liquid-graphite paste electrodes for the detection of 2,4,6-trinitrotoluene (TNT). Wang et al. ${ }^{75}$ have developed a facile strategy to dope graphene into layered doubled hydroxides (LDH). In doing so they were able to combine the adsorption and high catalytic activity of LDH with the excellent electrical conductivity of graphene. The stable graphene-LDH modified GC electrodes were used to enhance the determination of dopamine. Furthermore, the simple dispersion and dropcast method could easily be applied to enhance the conductivity of other nonconductive solids. Dispersion of CNTs in an inert matrix is one configuration that is particularly attractive for electroanalytic studies due to the increased mechanical robustness of the resulting electrode. In order to optimize this type of electrode, Olive-Monllau et al. ${ }^{76}$ studied the effect of various ratios of CNT to matrix. Their results demonstrated an optimal loading of 9-11\% MWCNTs, which was then utilized as a proof of concept in the electroanalytical detection of ascobic acid.

\section{Simultaneous Determination}

The ability to simultaneously determine multiple analytes of interest quickly, sensitively, and selectively make voltammetric analysis highly desirable for practical applications. Crucial to this application is the ability for the sensor to provide peak separation as well as providing enhanced sensitivity to the analytes of interest. A variety of materials, including carbon-based materials mentioned in the previous section, have been utilized to improve one or both of these properties in the papers discussed below.

The simultaneous determination of uric acid, ascorbic acid, and/or catecholamines is of particular interest due to the presence of these molecules in physiologically relevant specimens. Atta et al. ${ }^{77}$ have developed a composite film composed of palladium 
nanoclusters and poly (N-methylpyrrole) to modify a platinum electrode. The method of polymerization was found to be an important factor for achieving optimum sensing ability. The resulting sensor was then utilized for the simultaneous determination of a catecholamine, uric acid, and ascorbic acid. As a proof of concept the method was applied to pharmaceutical products, urine, and serum samples. Noroozifar et al ${ }^{78}$ prepared silver hexacyanoferrate nanoparticles which were then deposited on a CNT modified GC electrode to simultaneously determine ascorbic acid, dopamine, and uric acid. The modification of a GC electrode surface with CNTs and silver hexacyanoferrate nanoparticles improved both the resolution of the oxidation peaks and the catalytic activities toward the analytes under investigation. Ulubay and Dursun ${ }^{79}$ were able to perform simultaneous determination of dopamine and uric acid by incorporating copper nanoparticles with a polypyrrole modified GC electrode. The increased electrocatalytic activity towards the oxidation of these analytes was attributed to the increase in electronic conductivity and effective surface area.

Kalimuthu and John ${ }^{80}$ have used an ultrathin electropolmerized film of 2-amino-1,3,4thiadiazole to modify GC to simultaneously analyze ascorbic acid, dopamine, uric acid, and xanthine in human urine samples. The use of the electropolymerized film enabled the needed separation in the aforementioned analytes to make quantitative measurements. Further improvements and development of electropolymerized films will provide sensitive and selective substrates by which multianalyte detection can be performed.

Multianalyte determination has also been demonstrated for other analytes. Rastakhiz et al. ${ }^{81}$ have reported the preparation of a 1-(3,4-dihydro-4-oxo-3-phenylquinazolin-2-yl)-4phenylthiosemicarbazide modified CNT paste electrode for the simultaneous determination of phenylhydrazine, hydrazine, and sulfite. Ghorbani-Bidkorbeh et al ${ }^{82}$ have developed a methodology for the simultaneous determination of tramadol and acetaminophen using a GC electrode modified with phenylsulfonate functionalized carbon nanoparticles. The resulting electrode, with a high specific surface area, was able to enhance the oxidation peak currents for these analytes. The sensor was then used to determine the amount of acetaminophen and tramadol in pharmaceutical and biological preparations. Ensafi et al. ${ }^{83}$ were able to demonstrate the simultaneous determination of 6-thioguanine and folic acid through the use of a MWCNT paste electrode modified with ferrocenedicarboxylic acid. Here they employed differential pulse voltammetry, which has a much higher current sensitivity and better resolution than cyclic voltammetry.

\section{Molecularly Imprinted Electrodes}

The molecular imprinting technique has become a popular and powerful method for replacing biological receptors with synthetic molecules in order to provide a rapid, inexpensive, sensitive, and selective sensor. Molecular imprinting typically involves the preassembly of template molecules and functional monomer and the subsequent copolymerization with cross-linking monomers. The removal of template molecules from the polymer matrix generates the recognition sites (cavities) complementary to the shape, size, and functionality of the template molecule.

Several strategies are possible for the production of molecularly imprinted electrodes. Xie et al. ${ }^{84}$ describe a surface molecular self-assembly strategy to modify a gold nanoparticle-GC electrode for the enhanced detection of the pesticide chloropyrifos. In their study, chloropyrifos molecules were used to form imprint sites in the polyaminothiophenol membranes at the surface of gold nanoparticles modified GC electrode. The resulting sensor had an increased in voltammetric response enabling a much lower detection limit for chloropyrifos. Gomez-Caballero et al. ${ }^{85}$ have demonstrated the ability to generate an MIP with great control over the polymer thickness using an electrochemical synthesis. As a proof of concept, they used this method to make a MIP tailored for dopamine detection. Hu et al. ${ }^{86}$ prepared an MIP, which was added on top of nanocomposite film consisting of MWCNT 
decorated with $\mathrm{Fe}_{3} \mathrm{O}_{4} @ \mathrm{SiO}_{2}$ core-shell nanoparticles. The MIP provided numerous selective binding sites for the target analyte (benzylpenicillin), while the nanocomposite film enhanced the electrochemical signals. Yang et al. ${ }^{87}$ have develop a protocol by which MIPnanoparticles were prepared using the self-assembly micellization of an amphiphilic copolymer in order to circumvent the limitations of typical MIP-nanoparticle based sensors, such as incompatibility with aqueous systems and rigidity. The photo-crosslinkable copolymer provided a high specific surface area, which allowed for more recognition sites on the sensor. This general method was demonstrated using glucose, for which it was both selective and reproducible; however this protocol could be easily adapted for a wide range of analytes. By combining a molecular imprinting technique and nanotechnology, Zhang et al ${ }^{88}$ have prepared a sensitive tolazoline sensor. They used $o$-aminothiophenol as the functional monomer, which provided not only covalent $\mathrm{Au}-\mathrm{S}$ bonds to the nanoparticles but also enabled recognition sites through hydrogen and $\pi-\pi$ stacking interactions with the analyte. The resulting amperometric sensor had high catalytic activity that could be attributed to the gold nanoparticles, while the porous MIP film provided plentiful selective recognition sites.

Different methods of detection have also become popular for a variety of reasons. For example, Gholivand and Torkashvand ${ }^{89}$ developed an MIP sensor in carbon paste for the detection of metronidazole. While the sensor fabrication was relatively standard, they utilized cathodic stripping voltammetry during analyte determination, significantly reducing the analysis time. This is the first report of stripping voltammetry being utilized with an MIP modified electrode. Alizadeh et al..$^{90}$ have synthesized MIPs that were incorporated into CPEs. They used square wave voltammograms to measure to concentration of TNT in water and soil samples. Li et al. ${ }^{91}$ have developed a MIP sensor for the detection of oxytetracycline based on the competition reaction between the template molecule and an enzymatic amplifier. Due to the stereoscopic hindrance effect of the enzymatic amplifier, the target analyte is prevented from interacting with the electrode resulting in a measurable current change following an isolation step. This new determination method enabled high sensitivity and selectivity for the target analyte with a low cost sensor. The use of molecularly imprinted electrodes provides a cheap and durable method to reproduce the selectivity seen in biosensors. Further development for the use of molecularly imprinted electrodes as sensors provides a promising avenue for research in the coming years.

\section{Other Papers of Interest}

In the past two years there have been a number of important papers, that do not fall into any of the previous categories. Such novel advances are often very important for advancing the field of voltammetric sensors as they open up new avenues and methods for future research. Here we try to incorporate such articles and describe their importance while emphasizing that many important articles may not be included due to the sheer number of articles published in this field.

While potentiometric measurements through thin glass membranes is well understood, the high ohmic resistance of glass has prevented it use in voltammetric measurements. Contrary to convention, Velmurugan et al. ${ }^{92}$ demonstrated that nanometer-thick layers of dry glass are permeable to water but not other electroactive species. Additionally, they discovered that the nanometer-thick glass could be nearly entirely converted to a hydrated gel. This hydrated gel enabled selective permeability to the encased platinum wire. Further modification of nanometer-thick glass surrounding an electrode may provide a basis for improved applications, particularly in biological systems where biofouling is a serious problem.

Determination of ion concentrations in various liquids is common for both quality control and biological analyses. Typically potentiometric ISEs are used for routine monitoring of 
ions. While voltammetry is a widely used technique for measuring electroactive ions, its use for nonelectroactive ions is rare. Thus the report by Zhang et al. ${ }^{93}$ on a new form of voltammetric ISE provides a novel platform that allows for rapid measurements, small sample volumes, and high selectivity. By combining the concepts behind ionophore based ion-selective electrodes and using water-immiscible organic solvents adhered to an electrode surface they developed voltammetric cation and anion sensors, though the anion sensor for $\mathrm{Cl}^{-}$was non-Nerstian.

The ability to differentiate between enantiomers is a difficult yet important process, particularly with the shift in pharmaceuticals toward single-enantiomer drugs over racemates. Typically NMR spectroscopy has been used to determine the composition of enatiomeric mixtures, however this method requires distinguishable NMR signals and high concentrations for accurate measurements. Mirri et al. ${ }^{94}$ have developed a method by which a chiral ferrocene amine was used in conjunction with voltammetry to electrochemically detect the difference between $98 \%$ and $90 \%$ ee of (S)-Binol, and thus the detection of $<5 \%$ amounts of (R)-Binol at a concentration of $10^{-5} \mathrm{M}$. This proof of concept demonstration provides a promising avenue for an electrochemical method for chiral sensing.

Previous attempts to immobilize tyrosine onto electrodes have resulted in limited enzymatic activity. By utilizing Langmuir Blodgett (LB) methods, Pavinatto et al. ${ }^{95}$ were able to successfully modify indium tin oxide (ITO) and platinum substrates with a mixed Langmuir film. The resulting biosensor retained $12 \%$ of its original activity. Alessio et al. ${ }^{96}$ demonstrated a sensing platform that utilized both specific and non-specific interaction by using mixed LB films of a phospholipid and phthalocynines. In a proof of concept study, they demonstrated the ability for the mixed LB films to detect catechol at high sensitivity and stability.

The use of micro- and nano- structured particles to increase the surface area of electrochemical sensors has led researchers to develop a variety of methods for their fabrication and modification with and on various electrodes. Urbanova et al. ${ }^{97}$ have recently reported a strategy to create macroporous antimony film electrodes based on the electrochemical deposition of the metal into the interstitial space of a colloidal crystal template. By using well-defined polystyrene spheres as the template, they were able to carefully control the diameter of the resulting antimony pores. The porous film was significantly more sensitive to both cadmium and lead than planar antimony electrodes.

During the fabrication of DNA biosensors the immobilization of the single stranded probe on the surface of the electrode dictates the performance of the resulting sensor. Numerous methods for immobilization have been utilized, with the use of nanoparticles becoming popular in recent years due to the unique characteristics of these particles. Sun et al. ${ }^{98}$ have utilized a blend of nanomaterials including chitosan, $\mathrm{V}_{2} \mathrm{O}_{5}$ nanobelts, and MWCNT for the immobilization of single stranded DNA on a carbon ionic liquid electrode. Each component of the electrode provides a particular advantage, which in combination provides a stable and sensitive biosensor. The ability to combine the unique properties of individual nanomaterials provides a new and exciting frontier for the formation of novel electrodes.

Jin et al. ${ }^{99}$ have developed a method for preparing a SWCNT-based three-electrode system on a glass substrate using photolithography. The resulting SWCNT film was activated using an optimized $\mathrm{O}_{2}$ plasma treatment. While not demonstrated, this electrode system can be easily functionalized with biomacromolecules to generate a cost-effective, highly sensitive biocompatible sensor for a variety of applications. 


\section{AMPEROMETRIC SENSORS}

During an amperometric measurement the working electrode, or sensor, is held at a constant potential while the current is monitored. The current is then related to the concentration of the analyte present. ${ }^{2,3}$ This sensing method is commonly employed in both biosensors and immunosensors, which will be discussed further in later sections. In this section we will focus on recent papers that utilize amperometric methods without employing biologically derived materials as the sensing mechanism. Of particular interest are the novel employment of the amperometric method, the use of new materials, and improvements in non-enzymatic glucose and hydrogen peroxide $\left(\mathrm{H}_{2} \mathrm{O}_{2}\right)$ sensors.

Amatore et al. ${ }^{100}$ have developed a triple potential-step chronoamperometric method enabling the simultaneous detection of reactive oxygen and nitrogen species released by immunostimulated macrophages. The strategy uses a single microelectrode to make successive measurements at multiple potentials. The use of multiple potentials allowed for the detection of various reactive oxygen and nitrogen species released by a single living macrophage during induced phagocytosis. This proof of principle study demonstrates the feasibility for applying this method to monitor time dependence and composition of analytes in a biologically meaningful setting.

A more typical amperometric strategy was used by Khairy et al., ${ }^{101}$ who described how an unmodified screen printed shallow recessed graphite microelectrode array could be employed for the low micromolar determination of nitrite. The disposable nature and low cost of screen printed microelectrode arrays make this method of detection particularly promising. The modification of these microelectrode arrays could potentially make this a powerful detection method for a number of different analytes. Colombo et al. ${ }^{102}$ used a microelectrode array of boron-doped nanocrystalline diamond to perform amperometric measurements with micrometer spatial resolution. The excellent electrochemical properties of the electrodes coupled with the transparency and high spatial resolution of the array makes this sensor attractive for numerous applications. A microneedle array of CPEs were used by Windmiller et al. ${ }^{103}$ to generate a minimally invasive biosensor. The $3 \times 3$ pyramidal microneedle structures were loaded onto metalized carbon paste transducer. The resulting array could be easily modified, as was demonstrated by the authors who added lactate oxidase to produce a lactate sensor.

\section{Novel materials/composites}

The use of new materials, especially nanomaterials, has become an increased area of research in electrochemical sensors. The incorporation of these nanomaterials in conjunction with one another to form novel composites is particularly interesting, as many of these materials have been found to have synergistic effects. The explorations described here both solve problems of inter-material compatibility and investigate how the materials interact to improve sensors based on an amperometric method of detection.

Habibi et al. ${ }^{104}$ have optimized a MWCNT modified carbon-ceramic electrode for the enhanced determination of uric acid. The facile and rapid procedure for preparing this uric acid sensor is reported. The ceramic nature of this electrode allows it to be easily regenerated with a reproducibility of $99 \%$. The low cost and simplistic nature of this electrode make it a promising strategy for incorporation in real world applications. CNTs were also used by Guo et al., ${ }^{105}$ who developed a sensitive amperometric sensor for tryptophan by modifying a GC electrode with gold nanoparticle decorated CNTs. The nanocomposite material demonstrated synergistic enhancement for the electrocatlytic activity toward the oxidation of tryptophan. 
Electrospun carbon nanofibers provide more edge sites on the outer wall than CNTs, which may lead to more facile electron transfer, better dispersion, and better wettability. Tang et al. ${ }^{106}$ have used electrospun carbon nanofibers to modify a CPE. Their study demonstrated that this simple electrode can quantitatively determine L-tryptophan, L-tyrosine, and Lcysteine using cyclic voltammetry and constant-potential amperometric methods. The low cost, facile construction, high sensitivity, and lack of pretreatment make this electrode appealing for future electrochemical sensing application, both with and without further modification. Liu et al. ${ }^{107}$ mixed electrospun carbon nanofibers with the ionic liquid 1butyl-4-methylpyridinium hexaflurophosphate to produce a novel composite electrode. The resulting sensor combined the advantages of the two separate materials: high voltammetric response and low background noise making it applicable for amperometric determination of nicotinamide adenine dinucleotide NADH.

Wang et al. ${ }^{64}$ have developed a sensing platform that takes advantage of the unique properties of graphene nanosheets. They modified a GC electrode with poly(sodium styrenesulfonate)-graphene materials which have a high specific surface area and high electrial conductivity to produce a amperometric hydrazine sensor with a linear range of 3$300 \mu \mathrm{M}$. Brownson and Banks ${ }^{108}$ compared the use of graphene vs. graphite based amperometric biosensors. They found that graphite exhibited superior electrochemical response due to the increased number of edge plane sites. Interestingly, the introduction of Nafion reverses this trend as it disrupts the continuous nature of graphene. This further highlights the importance of edge plane sites discussed in the Voltammetric Sensor section of this review.

Tang et al. ${ }^{109}$ developed a novel method for producing a polyaniline/poly(acrylic acid) composite which was utilized for the determination of ascorbic acid. They found that the amounts of polyaniline and poly(acrylic acid) could be easily controlled by the number of potential cycles during fabrication. The resulting composite film exhibited excellent electrochemical activity in neutral and basic solutions.

Tao et al. ${ }^{110}$ have developed a sensitive methanol sensor based on an electrode composed of palladium-nickel/silicon nanowires. The closely packed silicon nanowires stand vertically on the electrode surface, providing high aspect ratios as well as the ability to be integrated with silicon-based integrated circuits. The nanoscale effects of the Pd-Ni layer enhance the electron mobility between the electrode-solution interface as well as increase the kinetics of the electrode.

Using novel nickel-based nanocomposites, Sattarahmady et al. ${ }^{111}$ were able to determine acetycholine without the use of any specific enzyme or reagent. In their study, a variety of composites composed of Ni microparticles, Ni nanoparticles, Ni nanoshells, carbon microparicles, and/or Nafion were used to modify a CPEs. The nickel catalysts present in all the tested sensors enabled the electrocatalytic oxidation of acetylcholine. A composite composed of nanoshells of hollow Ni microspheres, carbon microparticles, and Nafion proved to have the highest performance, allowing for nanomolar detection of acetylcholine. This systematic evaluation of nanomaterials with a known catalytic effect provides a useful platform for future studies examining methods for replacing enzymatic materials with more stable materials.

\section{Non-enzymatic glucose and $\mathrm{H}_{2} \mathrm{O}_{2}$ sensors}

A rapid, inexpensive, and reliable glucose sensor has been sought after for various applications including clinical, ecological, and food monitoring. The use of glucose oxidase (GOx), a biological enzyme which oxidizes glucose into gluconic acid in the presence of oxygen, to modify electrodes for glucose sensing has been thoroughly investigated. 
Unfortunately, the catalytic activity of GOx is susceptible to environmental conditions such as temperature, humidity, $\mathrm{pH}$, and toxic chemicals. Additionally, sensors based on this enzyme suffer from high cost and poor stability. Toghill and Compton ${ }^{112}$ recently reviewed the merits and shortfalls of non-enzymatic electrochemical glucose sensors (295 citations). According to the review, advancement in this field may depend on research into new carbon-based materials.

Yang et al. ${ }^{113}$ have prepared a non-enzymatic glucose sensor based on a novel nanocomposite comprised of MWCNTs and copper nanocubes on a tantalum substrate. The resulting amperometric sensor was capable of monitoring blood glucose levels in diabetic and non-diabetic patients. Wang et al. ${ }^{114}$ have prepared a non-enzymatic glucose sensor using a novel $\mathrm{Cu}-\mathrm{CuO}$ nanowire composite. The reported sensor was able to provide low detection limits $(0.05 \mathrm{mM})$ with a fast current response. Beyond long-term stability, the sensor was also able to determine glucose levels in the presence of uric acid and ascorbic acid, common interfering compounds. Jiang et al. ${ }^{115}$ used MWCNT electrodes modified with $\mathrm{CuO}$ nanoparticles via magnetron sputtering to detect glucose without GOx.

Importantly, the sensor was highly resistant to chloride fouling and interfering compounds, a common problem in copper based sensors. They also demonstrated the utility of this amperometric sensor in human serum samples.

Copper was also found to be essential by El Khatib and Hameed, ${ }^{116}$ who chemically reduced $\mathrm{Cu}_{2} \mathrm{O}$ on oil-furnace carbon black (Carbon Vulcan XC-72) to prepare a glucose sensor. They found that the morphology of $\mathrm{Cu}_{2} \mathrm{O}$ nanoparticles was affected by the molar ratios of reducing agent and copper salt, which in turn impacted the characteristics of the resulting sensor. Xu et al. ${ }^{117}$ generated a glucose sensor using copper polyhedron-patterened nanostructures prepared using ionic liquid assisted solvothermal synthesis. They found that the unique morphology of the copper nanostructures coupled with the presence of ionic liquid on the surface of these particles resulted in enhanced current response to glucose.

While most non-enzymatic glucose sensors are based on copper materials, several recent reports have described the use of other metals to act as a catalyst. Chen et al. ${ }^{118}$ have used palladium nanoparticles to modify functional CNTs for non-enzymatic amperometric glucose sensing. The resulting sensor was sensitive to glucose in the presence of chloride ions as well as other common interfering molecules. The long-term stability of the electrode was attributed to the use of palladium nanoparticles as the catalytic material. Yang et al. ${ }^{119}$ utilized a cobalt oxide/hydroxide nanostructure deposited onto MWCNTs to generate a nonenzymatic glucose sensor. The composite material was synthesized by using an electrochemical reduction. The modified electrode significantly enhanced the current response of the sensor compared with the CNTs alone. Wang et al. ${ }^{120}$ have developed a nonenzymatic amperometric glucose sensor based on nickel hexacyanoferrate nanoparticles. By performing cyclic voltammetry in a solution containing $\mathrm{Ni}^{2+}$ and $\mathrm{Fe}(\mathrm{CN})_{6}{ }^{3-}$, they were able to modify a GC electrode with well dispersed spherical particles that provided a large surface area. The resulting sensor was able to sensitively and stabile detect glucose without the presence of an enzyme. The simple method to simultaneously create nanoparticles and modify an electrode surface makes this approach particularly attractive. Mu et al. ${ }^{121}$ found that nanonickel oxide could be used to modify a CPE to generate a non-enzymatic glucose sensor. Furthermore, they found that performing potential scanning up to high potentials in alkaline solutionn increased the amount of $\mathrm{Ni}(\mathrm{OH})_{2} / \mathrm{NiOOH}$ redox couple present within the sensor, resulting in a improved electrocatalytical properties. Importantly, this paper presents a simple method for activating the catalytic material present on the non-enzymatic sensor. Seo et al. ${ }^{122}$ utilized nanoporous gold for glucose detection. By changing the deposition charge during the fabrication of the electrode, the pore size could be easily controlled. They found that the dimensions of the pores in the gold were of great significance to the detection 
of glucose, with an optimized pore size of $\sim 16 \mathrm{~nm}$. Because gold electrodes can be easily modified, further investigations using this sensing material appear promising.

Nanoporous gold was also used by Meng et al. ${ }^{123}$ for the non-enzymatic detection of $\mathrm{H}_{2} \mathrm{O}_{2}$. The fact that this material can be used for both glucose and $\mathrm{H}_{2} \mathrm{O}_{2}$ detection make this material both highly promising as well as concerning from a selectivity point of view, however both papers ${ }^{122,123}$ describe the developed sensors as being highly selective. The production of $\mathrm{H}_{2} \mathrm{O}_{2}$ during chemical and enzymatic processes makes its quantitative detection extremely important for biosensors. Recent research has focused on the use of new materials for enhancing $\mathrm{H}_{2} \mathrm{O}_{2}$ sensing properties. The use of nanomaterials has generated much excitement in this area because their large specific surface area decreases the overpotential required to detect many analytes.

Lin et al. ${ }^{124}$ have modified a fluorine-doped tin oxide electrode with zinc oxide nanorods decorated with silver nanoparticles. The resulting sensor was able to selectively detect $\mathrm{H}_{2} \mathrm{O}_{2}$ in the presence of uric acid and ascorbic acid. Bo et al. ${ }^{125}$ reported a facile method for incorporating copper sulfide nanoparticles inside ordered mesoporous carbons. The resulting nanocomposite was then used to enhance the electrocatalytic activity towards $\mathrm{H}_{2} \mathrm{O}_{2}$. The fast response and simple preparation makes this nanocomposite promising for a nonenzymatic $\mathrm{H}_{2} \mathrm{O}_{2}$ sensor. Salimi et al.126 used CNTs modified with a phenazine derivative of manganese complex that is capable of a quasi-reversible $1 \mathrm{e}^{-} / \mathrm{H}^{+}$redox process over a wide $\mathrm{pH}$ range. The resulting $\mathrm{H}_{2} \mathrm{O}_{2}$ sensor had remarkable catalytic activity, good reproducibility, and a facile fabrication method.

Xu et al. ${ }^{127}$ have modified vertically aligned MWCNTs with manganese oxide $\left(\mathrm{MnO}_{2}\right)$ for a sensitive and stable non-enzymatic $\mathrm{H}_{2} \mathrm{O}_{2}$ sensor. The combination of catalytic $\mathrm{MnO}_{2}$ particles with the high surface area provided by the CNTs allowed for both sensitive and selective determination of $\mathrm{H}_{2} \mathrm{O}_{2}$, which was demonstrated in the routine analysis of $\mathrm{H}_{2} \mathrm{O}_{2}$ in milk. Using a coprecipitation method, Cui et al. ${ }^{128}$ were able to prepare $\mathrm{Cu}-\mathrm{Mg}$-Al calcined layered double hydroxides that were used to modify a GC electrode. The layered double hydroxide promoted the electron transfer between $\mathrm{H}_{2} \mathrm{O}_{2}$ and the underlying electrode enabling efficient $\mathrm{H}_{2} \mathrm{O}_{2}$ detection.

Salazar et al. ${ }^{129}$ have demonstrated the use of Prussian Blue-modified carbon fiber microelectrodes as an alternative for $\mathrm{H}_{2} \mathrm{O}_{2}$ detection in brain extracellular fluid. They deposited the Prussian Blue film on carbon fiber electrodes using electrodeposition, which were then activated using cyclic voltammetry. The modified electrode could then be further modified to produce a sensitive biosensor that requires very low potentials to avoid biofouling and interference problems. Prussian Blue was also used by Jiang et al., ${ }^{130}$ who electrodeposited the material onto graphene to sensitively detect $\mathrm{H}_{2} \mathrm{O}_{2}$ and hydrazine. They attributed the enhanced electrocatalytic activity of the resulting sensor to a synergistic effect between graphene and Prussian Blue.

\section{IMMUNOSENSORS}

Immunosensors, which perform immunoassays based on antigen and antibody recognition, have become vital for the determination of biochemical targets relating to health concerns spanning from cancer antigens in patient serum to bacterial species in food. ${ }^{2}$ A continual concern with immunosensor development is the capability to sensitively detect relevant immunological compounds without compromising the bioactivity of the immunoactive species on the electrode. Hartwell et al. ${ }^{131}$ published a review on recent trends in flow-based immunosensors (234 citations). Many researchers have increasingly utilized nanomaterials to support the immunoactive agents while simultaneously enhancing the electrochemical and analytical capabilities of the electrode. Here, we focus on the use of CNTs for electrode 
fabrication, trends in cancer detection using $\alpha$-fetoprotein and carcinoembryonic antigen, and sandwich-type electrodes for amplification of signals.

\section{Carbon Nanotubes}

CNTs play an important role in recent trends for immunosensor fabrication. Immobilization of the bioactive species is crucial for proper detection and CNTs offer an easy way to protect and stabilize these species. In addition to other nanomaterials, these composite immunosensors have exhibited good analytical characteristics and have shown great promise for clinical applications. Jacobs et al. ${ }^{57}$ have published a review focusing solely on biomolecule detection using CNT-based sensors (202 citations). Cancer detection has also benefitted from the inclusion of CNTs for immunosensors. Ji et al. ${ }^{132}$ published a review highlighting recent advances in cancer research geared toward rapid diagnosis and potential therapeutics (84 citations).

In addition to cancer detection, CNTs have allowed for novel immunosensor development to advance screening capabilities for biomolecules. For example, Serafín et al. ${ }^{133}$ recently developed a clinically relevant immunosensor for testosterone. Due to the illicit use of testosterone agents to boost athletic ability and its subsequent health concerns, improved sensing capabilities are necessary. These researchers found that a combination of gold nanoparticles, MWCNTs, and Teflon allowed for a superior electrode composition ensuring the stability of the immunoactive agent, which were monoclonal antitestosterone antibodies. The resulting immunosensor could easily measure testosterone from serum with little preparation.

Electrochemiluminescence has become increasingly popular in analytical chemistry due to its variety of applications and relative ease of use. Jie et al. ${ }^{134}$ recently reported their novel electrochemiluminescence immunosensor using CdS quantum dots and CNTs working in tandem with gold nanoparticles-chitosan. Their sensor enabled successful antibody immobilization and held exemplary analytical characteristics for biological environments.

Two recent examples of CNT-based immunosensors for cancer biomarkers revolve around interleukin- 6 and $\alpha$-fetoprotein. Interleukin- 6 is involved in immunological processes that can lead to squamous cell carcinomas. To enhance detection of interleukin-6, Malhotra et al. ${ }^{135}$ designed and tested a sensor composed of a SWCNT forest platform with multilevel capture antibody functionalization. This novel immunosensor correlated well with enzymelinked immunosorbent assays and performed successfully in a variety of physiological appropriate concentrations.

\section{Cancer Detection}

Accurate detection of cancer can be a clinical challenge due to the lack of approved and applicable serum tests for specific cancer types. Immunosensors are becoming increasingly utilized for cancer detection due to their inherent specificity and accuracy. Additionally, an improvement of sensitivity in cancer detection could lead to earlier intervention and successful treatments. CNTs were successfully used in an $\alpha$-fetoprotein sensor developed by Che et al. ${ }^{136}$ using GC electrodes with surface modification to include MWCNTs and chitosan- $\mathrm{MnO}_{2}$. This modified electrode housed anti- $\alpha$-fetoprotein, further immobilized by gold nanoparticles. The composition of the electrode provided excellent conductivity, improved performance, and successfully amplified the signal. The successful characterization and use of this novel immobilization technique provides evidence of its practical application for the detection of other proteins of interest.

One commonly used biomarker for diagnosis and treatment of cancer is $\alpha$-fetoprotein, but unfortunately current clinical $\alpha$-fetoprotein serum tests are unreliable. Development of an $\alpha$ - 
fetoprotein sensor could enhance diagnostic ability and lead to better treatment choices for patient care. To ensure rapid, accurate detection of $\alpha$-fetoprotein in serum, Giannetto et al. ${ }^{137}$ developed a sandwich-type nanocomposite and GC electrode immunosensor. Monomeric thionin was utilized in the reading solution to avoid immobilization issues while providing ease of fabrication. This novel electrode exhibited good analytical characteristics in a variety of samples when compared with enzyme-linked immunosorbent assay. This method of fabrication allows for reproducibility, ease of fabrication and use, and storage stability enabling potential clinical use for rapid $\alpha$-fetoprotein detection. Du et al. ${ }^{138}$ also constructed an immunosensor to detect and amplify the signal for $\alpha$-fetoprotein. The immunosensor was constructed using labeled carbon nanospheres and a graphene sheet platform. The nanospheres were capable of binding to multiple horseradish peroxidasesecondary antibodies as well as its bioconjugates, thus enhancing sensitivity. The graphene sheet platform increased the surface area and aided in the amplification of detection response. An alternate immunosensor for $\alpha$-fetoprotein detection was developed by Tang et al. ${ }^{139}$ and showed promising results when testing clinical serum samples. The trace label was made by using labeled horseradish peroxidase-anti- $\alpha$-fetoprotein conjugates on irregularly shaped gold nanoparticles, allowing for a low detection limit and good analytical characteristics. For an immunosensing probe, functionalized biomimetic carbon nanoparticles were used, which aided in immobilization and electron transfer. Using graphene sheets, Wei et al. ${ }^{140}$ developed a novel label-free immunosensor for $\alpha$-fetoprotein detection. This method immobilized anti- $\alpha$-fetoprotein onto the graphene sheets and a modified GC electrode. Analytical characteristics were measured by cyclic voltammetry and provided reliable results. This label-free immunosensor is easier to fabricate than alternate sandwich-type sensors, allowing for ease of production and makes it readily suitable for clinical use.

Diagnostics have also been aided by the development of immunosensors specific to carcinoembryonic antigen, which is over-expressed in serum samples of certain cancer types, thus making it a reliable biomarker for clinical diagnosis. Magnetic nanoparticles have become a useful tool in carcinoembryonic antigen immunosensor development. Li et al. ${ }^{95}$ functionalized gold/ $\mathrm{Fe}_{3} \mathrm{O}_{4}$ nanoparticles with thiourea, which could then directly immobilize carcinoembryonic antibodies. These magnetic particles were then attached to a solid paraffin CPE platform, making the electrode easy to fabricate and able to regenerate. Their studies indicated that the analytical characteristics of their novel immunosensor are superior to alternative techniques and require less money and time to construct, making this extremely useful for clinical applications with tumor detection. Laboria et al. ${ }^{141}$ have developed a novel class of bipodal thiolated self-assembled monolayers that contain reactive $\mathrm{N}$-hydroxysuccinimide ester end groups that were used to immobilize anti-carcinoembryonic antigen monoclonal antibody. The resulting amperometric immunosensor was capable of detecting carcinoembryonic antigen, a tumor marker in cancer patients, in serum samples from colon cancer patients. The use of these self-assembled molecules may provide a platform for the simple immobilization of numerous antibodies, making multiplexed detection of biomarkers possible. Liao et al. ${ }^{142}$ have developed a novel composite material composed of Nafion and cysteine, enabling the covalent attachment of gold nanoparticles, which were further modified carcinoembryonic antibody to form an amperometric immunosensor. The large specific surface area, biocompatibility, and electronic properties of gold nanoparticles have lead to their use in numerous biosensors. Thus the compact, stable, biocompatible, and electron transfer ability of the Nafion/cysteine composite provides an attractive alternative for the immobilization of functionalized gold nanoparticles in a variety of potential sensing applications. Song et al. ${ }^{143}$ have described a fabrication method for an immunosensor based on the immobilization of an antibody on a redox biocompatible composite membrane. The composite membrane was composed of gold nanoparticles doped chitosan-MWCNTs modified with Prussian Blue nanoparticles and gold nanoparticles. The 
simple methodology provides a stable three-dimensional structure that is biocompatible and can be easily modified with antibody molecules for use in a variety of immunosensors. As a proof of concept, they immobilized anti-carcinoembryonic antigen to demonstrate the superior sensitivity and sensing activity of the construct. Carcinoembryonic antigen has also been detected by a novel immunosensor fabricated by Zhong et al. ${ }^{144}$ by using a variation on the sandwich-type immunoassay. For trace labels their sensor used nanogold-enwrapped graphene nanocomposites. These labels were attached by electrochemical deposition onto a GC electrode via a Prussian Blue coating. Serum samples were tested and confirmed with reference values, suggesting the use of this sensor for rapid determination in a clinical setting.

\section{Sandwich-Type Sensors}

Sandwich-type immunosensors, or two site-type immunosensors, are considered to provide a more sensitive platform. In these immunosensors one antibody is used to attach the antigen to a solid matrix, while a second antibody is used to carry the detection system; typically an enzyme. Sandwich-type immunosensors have becoming vital for the detection of biomolecules due to the ability of the sensor to rapidly amplify responses. In the past two years, sandwich-type sensors have been fabricated for a multitude of applications including cancer detection and bacterial contamination, making this type of sensor greatly applicable to health, environmental, and food industries.

Li et al. ${ }^{145}$ demonstrated a facile strategy for preparing sandwich-type immunosensors based on magnetic mesoporous nanomaterials. A combination of organic and inorganic nanomaterials were used to facilitate both absorption and performance of the sensor. As a proof of concept, they immobilized anti-carcinoembryonic antigen and horseradish peroxidase to generate a sensitive immunosensor for the detection of carcinoembryonic antigen.

In the health industry, pancreatic cancer is difficult to diagnose in its early stages and extremely lethal in later stages, when it is more diagnosable. Carbohydrate antigen 19-9 is a popular and preferred label for pancreatic cancer and has become widely used in immunosensor development. Gu et al. ${ }^{146}$ utilized $\mathrm{ZnO}$ quantum dots as labels in a sandwichtype immunosensor constructed by functionalizing Si substrates and performing immunoreactions of antibodies and antigens to successfully detect carbohydrate antigen 19-9. The resulting immunosensor exhibited good analytical characteristics as well as promising results for amplification of response, stability over time, and uniformity; indicating that variations on the composition and type of antigens used could lead to sensitive immunosensors for other diseases. Quantum dots have also been utilized by Yang et al. ${ }^{147}$ to functionalize graphene sheets to prepare a sandwich-type immunosensor, where anti-prostate specific antigen was used as the primary antibody. The quantum dot functionalized graphene served to amplify the signal of the antibody.

Widespread use of the vital biomarker, tumor necrosis factor- $\alpha$ (TNF- $\alpha$ ), has afforded researchers the opportunity to examine a multitude of biological processes. Recently, Yin et al. ${ }^{148}$ developed a sensitive, novel sandwich-type immunosensor for the detection of TNF- $\alpha$. They used a poly (styrene-acrylic acid) surface to assemble gold nanoparticles as a composite matrix for alkaline phosphatase, which could then be used as a label. Immobilization of TNF- $\alpha$ occurred at a modified GC electrode, enabling the sandwich-type immunoreaction.

Food analysis has always been vital in industry to ensure the lack of contaminants such as staphylococcal enterotoxin B, which causes symptoms related with food poisoning and is thought to be a biological warfare threat. A novel sandwich-type immunoassay developed by 
Tang et al. ${ }^{149}$ has been successful in detecting a dynamic range of staphylococcal enterotoxin B contamination in doped food samples. Their immunosensor relies on polyclonal anti-staphylococcal enterotoxin B antibodies adhered to a screen-printed carbon electrode. This electrode is then bound to a MWCNT doped with horseradish peroxidasenanosilica, which aided in amplification of the signal.

Ahirwal et al. ${ }^{150}$ recently developed an electrochemical immunosensor using gold nanoparticles and an attached antibody. They investigated the sensing capabilities with cyclic voltammetric experiments and also evaluated it using electrochemical impedance spectroscopy. The resulting data indicated that this sandwich-type immunosensor allowed for antibody stability and adequate sensitivity, suggesting its potential application for immunoassays. Gold nanoparticles were also used by Wang et al., ${ }^{151}$ who incorporated nanomaterials and antibodies to develop a sensitive sandwich-type electrochemical immunosensor. The sensing ability was derived from horseradish peroxidase-anti-human immunoglobulin $\mathrm{G}$ that was adsorbed on $\mathrm{Au} / \mathrm{SiO}_{2}$ nanoparticles. The stable and biocompatible nanoparticles provided a large specific area for the secondary antibody layer. The amperometric response was based on the catalytic reduction of $\mathrm{H}_{2} \mathrm{O}_{2}$ at the gold nanoparticle/polythionine modified GC electrode that formed the bottom of the sandwich immunosensor. This strategy, which utilizes the advantages of both biomaterials and nanomaterials, may be extended to other bio-hybrid devices for bioanalytical and bioseperation applications.

The advantages of nanomaterials have also warranted their use in other sandwich-type immunosensors for the detection of neomycin and human Immunoglobulin G (IgG). Using this sandwich approach, Zhu et al. ${ }^{36}$ have developed a sensitive neomycin immunosensor. The sensor was based on the combination of a new conducting polymer, poly-[2,5-di-(2thienyl)-1H-pyrrole-1-(p-benzoic acid)], and a hydrazine conjugate. The conducting polymer was used to covalently immobilize the primary antibody, while the hydrazine/ MWCNT/gold nanoparticle conjugate enabled an accurate amperometric response. Additionally, they demonstrated how the deposition of gold nanoparticles onto the CNTs produced an enhanced response, enabling the measurement of neomycin in real sample analysis. Yang et al. ${ }^{152}$ have developed an ultrasensitive electrochemical immunosensor utilizing labels based on mesoporous silica nanoparticles that are capable of simultaneous loading of mediator and enzyme, while also serving as a carrier for the secondary antibody. The sandwhich-type protocol was based on covalent attachment of various molecules to the mesoporous silica nanoparticles. The synergistic effects of the resulting immunosensor was $\sim 100$ times more sensitive to human IgG than sensors missing a component of the sandwich (mediator or enzyme), demonstrating the importance of having both components.

Su et al. ${ }^{153}$ demonstrated how gold-silver-graphene hybrid nanosheets could be used to enhance the sensitivity of a sandwich-type immunosensor. The $\alpha$-fetoprotein sensor used the hybrid nanosheet to both increase the loading capacity and improve the electrochemical properties of the immunosensor. AuTi nanolabels were used to amplify the electrochemical signal, resulting in a sensor capable of detecting $\alpha$-fetoprotein with a detection limit of 0.5 $\mathrm{pg} / \mathrm{mL}$.

\section{Other Papers of Interest}

Toxicological studies have relied heavily on miniscule detection of bioactive agents. Whether the toxin being studied is purposefully administered as a therapeutic or accidentally introduced as a contaminant, successful determination of the toxin is vital for treatment options. One such potentially toxic biomolecule is salbutamol. It is currently used as a bronchodilation induction agent for therapeutic relief from a variety of respiratory pathway diseases. Unfortunately, residues of salbutamol agents can be readily found in consumable 
meats and are toxic to humans, necessitating a selective immunosensor for minute salbutamol quantity detection. Current detection techniques are time consuming, require highly trained technicians, and expensive. Huang et al. ${ }^{154}$ recently reported the use of gold nanoparticles with horseradish peroxidase-anti salbutamol as labels for their novel polyaniline/poly (acrylic acid) nanocomposite immunosensor. The resulting analytical characteristics were superior to alternative techniques when considering stability over time, accuracy, reproducibility, and ease of fabrication.

Another biomolecule of interest is somatomedin C, or insulin-like growth factor-1. Somatomedin $\mathrm{C}$ is becoming increasingly important in research for a variety of diseases from hearing loss to cancer. Rezaei et al. ${ }^{155}$ reported their use of gold nanoparticles and monoclonal antibodies on modified gold electrodes for novel detection of insulin-like growth factor-1. Cyclic voltammetry and electrochemical impedance spectroscopy ensured proper function of the immunosensor and preliminary results suggest its effective detection in human serum samples.

Detection of cholera toxin is vital due to its capability to promote severe illness, death, and potential use in biological warfare. Gold nanoparticles have been recently used by Loyprasert et al. ${ }^{156}$ to develop an extremely sensitive cholera toxin immunosensor by absorbing anti-cholera toxin onto the nanoparticles then attached to a polytyramine-modified gold electrode. This sensor had superior detection to alternative sensors and exhibited valid measurements in water samples.

Currently, detection of sulphate-reducing bacterium can take weeks to complete. To ensure rapid detection of this bacterium, Wan et al. ${ }^{157}$ have developed a novel impedimetric immunosensor using reduced graphene sheets doped with chitosan nanocomposite films. This immunosensor exhibited good analytical characteristics, biocompatibility, and a novel architecture suitable for the immobilization of various types of biochemical targets.

Recent trends for immunosensor development have focused on the miniaturization of the sensors design by incorporating nanomaterials. Variations on the nanomaterials utilized have also aided in electron transfer, bioactive agent immobilization, and stability, thus producing reliable sensors. Based on these current publications, immunosensor development is moving toward mobile devices for the rapid detection of targeted species. This will enhance clinical diagnostics and eventually lead to custom therapies for illnesses that are currently not adequately detectable in early, treatable stages.

\section{BIOSENSORS}

Biosensors aim to utilize the power of electrochemical techniques for biological processes by quantitatively producing an electrical signal that relates to the concentration of a biological analyte. ${ }^{2}$ The relatively low cost and rapid response of these sensors make them useful in a variety of fields including healthcare, environmental monitoring, and biological analysis among others.

There have been a number of recent review articles that have focused on the development of various materials, techniques and applications of biosensors. A review by Wang et al. ${ }^{158}$ that focused on the use of graphene in direct electrochemistry of redox enzymes, enzyme based biosensors and the electrocatalytic detection of small molecules (137 citations). The use of zinc-oxide based enzymatic biosensors and their fabrication are covered by Zhao et al ${ }^{159}$ (56 citations). Siqueira et al. ${ }^{160}$ provide an overview of the use of nanostructured films in biosensors (132 citations). Harper and Anderson ${ }^{161}$ reviewed the developments in using CNTs and electrostatic assembly in glucose sensors (133 citations). Also, there are reviews based on specific techniques including a review by Singh et al. ${ }^{162}$ that focused on the 
developments in peptide nucleic acid in DNA biosensors (108 citations) while Park et al. ${ }^{163}$ covered progress in techniques using encapsulated enzymes (102 citations). A review by Su et al. ${ }^{164}$ described the advances utilizing microorganisms to measure analytes (137 citations). Since there have been a wealth of biosensor developments in the past two years, we primarily focused giving an overview of new approaches and materials for enzyme based sensors and DNA sensors.

\section{Enzyme-Based Biosensors}

Enzyme based electrodes require the immobilization of an enzyme onto an electrode surface for the quantification of an analyte and are receiving increased attention due to potential applications in clinical, environmental and manufacturing areas. ${ }^{2}$ Recent development has focused on improving the immobilization and stability of the enzymes.

Glucose Sensors-Glucose sensors have been extensively studied because of the importance of monitoring blood glucose in diabetics. To improve upon current sensing methods, nanostructured materials are being used to immobilize enzymes. The nanostructure provides high catalytic activity and high surface affinity. ${ }^{159,165} \mathrm{Li}$ et al. ${ }^{165}$ developed a glucose biosensor, which uses $n$-alkylamine stabilized palladium nanoparticles to immobilize GOx onto a modified GC electrode, which provided a hydrophobic layer preventing enzyme leaching. When tested, these electrodes were accurate and precise at determining blood glucose in human blood serum and retained $90 \%$ of selectivity after three-months of storage.

A thin-walled graphitic nanocage material with well-developed graphitic structure was synthesized by Guo et al. ${ }^{166}$ to provide a sensing interface for a glucose biosensor. The authors determined the specific surface area to be as high as $613 \mathrm{~m}^{2} \mathrm{~g}^{-1}$, significantly higher than other carbon-based materials such as CNTs. They coupled this material with GOx to generate an amperometric glucose sensor. The unique physicochemical properties of this material, including the high specific surface area and pronounced mesoporosity, make it a suitable platform for a number of electrochemical sensors.

Ahmad et al. ${ }^{167}$ have demonstrated how a single zinc oxide $(\mathrm{ZnO})$ nanofiber can be fabricated and modified with GOx to form a highly sensitive amperometric glucose biosensor. The electrospun $\mathrm{ZnO}$ nanofibers were deposited onto a gold substrate with the aid of poly(vinyl alcohol). The hydrophobic nanofiber provides a favorable environment for the enzyme, making it an ideal platform for increasing the stability of the resulting biosensor.

Baby and Ramaprabhu ${ }^{168}$ have used silicon dioxide coated magnetic nanoparticle decorated CNTs as an electrocatalytic platform for GOx. The resulting glucose biosensor displayed high sensitivity and a wide linear range that makes this sensor potentially useful in glucose determination in food industries. In a one-pot chemical synthesis, Zou et al. ${ }^{169}$ prepared a novel magnetic polymeric bionanocomposite with GOx immobilized at a high load while retaining high activity. The magnetic activity of the composite allowed for simple and efficient separation and immobilization onto a gold magnetism-electrode to form a glucose biosensor.

Tang et al. ${ }^{170}$ have used an electrospinning technique to deposit $\mathrm{TiO}_{2}$ nanofibers on a platinum electrode. The resulting substrate had excellent electrocatalytic activity, showing a $30 \%$ increase in current density response to $\mathrm{H}_{2} \mathrm{O}_{2}$ than a bare platinum electrode. The authors then immobilized GOx with the aid of chitosan to provide a high performance glucose sensor. The electrospun $\mathrm{TiO}_{2}$ nanofibers provide a promising platform for further investigation in electrochemical biosensors. 
Wang et al. ${ }^{171}$ used a chitosan-stabilized hollow nanostructured platinum decorated with MWCNTs to drive the direct electron transfer between GOx and the surface of a GC electrode and positive poly(diallydimethylammunium) chloride protected gold nanoparticles to create a better support and improve immobilization of GOx. The resulting electrode had a lower limit of detection and a larger linear range compared to other MWCNTs and also retained $83.7 \%$ of its initial current response after three weeks.

Wan et al. ${ }^{172}$ have developed a method for the covalent attachment of glucose oxidase (GOx) within a three-dimensional surface network structure. The method involves modifying a gold electrode with a self-assembled monolayer upon which polyaniline and chitosan-coupled CNTs could be added as the signal amplifiers for the GOx detecting molecules. The covalent attachment platform prevents GOx from leaching, providing a more stable biosensor with high sensitivity and selectivity.

Another nanomaterial used to improve immobilization and selectivity of GOx was silicon nanowires (SiNWs). SiNWs are a popularly employed nanomaterial because of their unique physical properties and their ability to be mass produced, which makes them ideal for diagnostic tests. Su et al. ${ }^{173}$ used SiNWs decorated with gold nanoparticles (SiNWs@AuNPs) to support direct electrochemistry of GOx on GC electrodes. They found that SiNWs@AuNPs alone had a relatively poor limit of detection, $500 \mu \mathrm{M}$, however when Nafion was added there was a tenfold improvement. Also, they determined that when testing is done in the presence of interfering substances there was no change in ampermeric current. Murphy-Perez et al. ${ }^{174}$ used vapor-liquid-solid grown silica nanowires $\left(\mathrm{SiO}_{2} \mathrm{NWs}\right)$ to covalently immobilize GOx showing higher enzyme loading and linearity in the range of interest.

Zhang et al. ${ }^{175}$ have investigated a surface-initiated atom-transfer radical polymerization method for developing an enzyme-mediated amperometric biosensor. Using a coupling agent containing an atom-transfer radical polymerization initiator, the authors were able to modify an ITO electrode with ferrocenylmethy methacrylate and glycidyl methacrylate. GOx was then immobilized on the electrode through coupling between the epoxide groups of glycidyl methacrylate and the amine groups of GOx. The authors discovered that the use and order of the block copolymer provided a spatial effect that enhanced sensitivity to glucose.

Lactate Sensors-The quantification of lactate is also becoming increasingly more important for clinical purposes for the diagnosis of diseases including tissue hypoxia, respiratory and renal failure. Romero et al. ${ }^{176}$ developed a lactate biosensor in which the platinum surface is protected by a Nafion membrane, with lactate oxidase (LOx) immobilized in a mucin/albumin matrix on the top and a final protecting Nafion layer on the surface. The design enabled this sensor to be used in whole blood with improved selectivity and sensitivity. The sensor kept a relatively unchanged sensitivity for five months with a detection limit of $0.8 \mu \mathrm{M}$, and when assay were performed with whole blood there was a recovery of $89 \pm 6 \%$.

Other modifications on lactate sensors have been used to expand the linear range, create novel immobilization protocols and fabricate electrogenerated chemiluminescense. ${ }^{177-179}$ Gamero et al. ${ }^{177}$ on lactate sensors consist of immobilizing onto a rough gold electrode modified with a self-assembled monolayer of dithiobis-N-succinmidyl propionate (DTSP), which allows for a larger electrocatalytic activity and linear range, up to $1.2 \mathrm{mM}$. The novel enzyme immobilization protocol utilizing hydrolysis of alkoxysilanes created a more active and stable membrane. ${ }^{178}$ Electrogenerated chemiluminescense was obtained by MWCNTs that were decorated with $\mathrm{ZnO}$ nanoparticles immobilized onto GC electrodes further 
modified with LOx and Nafion creating an electrogenerated chemiluminescense sensor that was tested using human blood plasma. ${ }^{179}$

Other Enzymatic Sensors-Other vital analytes that can be quantified by enzymatic biosensors are $\mathrm{H}_{2} \mathrm{O}_{2}$, copper compounds and polyphenols. In recent work by Sheng et al.180 they describe the use of heme-proteins immobilized in porous carbon nanofiber/RTIL film for the detection $\mathrm{O}_{2}, \mathrm{H}_{2} \mathrm{O}_{2}$, and $\mathrm{NO}$ reduction. Safavi and Farjami ${ }^{181}$ developed a novel hydrogen peroxide amperometric sensor that uses a composite film of ionic liquids to immobilize myoglobin on a GC electrodes and has a lower detection limit than comparable heme sensors. In two seperate papers by Akyilmaz et al. ${ }^{182,} 183$ they describe the development of a tyrosine enzyme sensor and a partially purified polyphenol oxidase enzyme sensor for the catechol oxidase. Also, Fusco et al. ${ }^{184}$ developed a polyphenol sensor using laccases immobilized on MW- and SW-CNTs screen-printed electrodes for the detection of polyphenols in wine, commonly called tannins, which have well known antioxidant properties.

Nanomaterials have also been utilized to enhance the detection of $\mathrm{H}_{2} \mathrm{O}_{2}$, a compound of particular importance in biological systems. Lu et al. ${ }^{185}$ have prepared a $\mathrm{MgO}$ nanoparticlechitosan composite matrix to immobilize horseradish peroxidase forming a sensitive $\mathrm{H}_{2} \mathrm{O}_{2}$ biosensor. The chitosan was utilized to form a biocompatible interface between the catalytic $\mathrm{MgO}$ nanoparticles and the enzyme. The resulting sensor had a high specific surface area, high sensitivity and fast response towards $\mathrm{H}_{2} \mathrm{O}_{2}$ in the presence of hydroquinone as a mediator.

Using a hydrothermal technique, Kafi et al. ${ }^{186}$ have developed a method for the growth of nanoporous gold networks on titanium substrates. The large surface area of the supporting matrix was used to immobilize hemoglobin to produce a high-performance $\mathrm{H}_{2} \mathrm{O}_{2}$ biosensor. The nanoporous gold network significantly enhanced the sensing ability when compared to a titanium substrate modified with planar gold.

Ding et al. ${ }^{187}$ used an electrospinning technique to directly deposed highly porous hemoglobin microbelts onto a GC electrode surface without using an immobilization matrix. They used several spectroscopic methods to demonstrate that the hemoglobin kept its native structure within the microbelt morphology. The resulting electrode was used to detect both $\mathrm{H}_{2} \mathrm{O}_{2}$ and nitrite. This matrix- and mediator-free sensor fabrication process is very intriguing for direct electrochemistry-based biosensors.

Ndangili et al. ${ }^{188}$ have described the formation of nanofibrillar polyaniline-polyvinyl sufonate composite through the electropolymerization of aniline in the presence of ferrocenium hexafluorophophate. They then used the composite to absorb horseradish peroxidase to form an amperometric enzyme biosensor. This polymer based-substrate provides a promising platform for mediated-redox-enzyme amperometric biosensors.

Sheng et al. ${ }^{180}$ were able to immobilize heme-proteins in a composite material composed of porous carbon nanofiber and a room-temperature ionic liquid. The composite material provided a microenvironment enabling the heme-proteins to retain their native structure. The resulting biosensor was capable of detecting $\mathrm{O}_{2}, \mathrm{H}_{2} \mathrm{O}_{2}$, and NO.

Won et al. ${ }^{189}$ have used a $\mathrm{Fe}_{3} \mathrm{O}_{4}$ nanoparticle core-mesoporous silica shell matrix to immobilize horseradish peroxidase to develop a $\mathrm{H}_{2} \mathrm{O}_{2}$ biosensor. The mesoporous surface of the silica shell provides biocompatible entrapment sites, while the nanoparticle structure increases the surface area and loading capacity. The resulting biohybrid material was deposited onto screen-printed electrodes providing a sensitive biosensor. 


\section{Direct Electron Transfer}

Typical biosensors utilize natural or artificial redox mediators to monitor an enzymatic reaction that is both selective and sensitive for a particular analyte. Yet an ideal approach would allow for direct electron transfer between the enzyme and the electrode in order to increase selectivity, simplify the process, and requiring fewer reagents. Unfortunately enzyme immobilization remains a challenge for biosensors based on direct electron transfer. Novel immobilization methods are thus a crucial step for advancing this field.

Silveira et al. ${ }^{190}$ have developed a protocol by which cytochrome c nitrite reductase was incorporated within a porous sol-gel matrix to modify a pyrolytic graphite electrode. The resulting non-mediated amperometric biosensor demonstrated enhanced selectivity toward nitrite. Abdelwahad et al. ${ }^{191}$ were able to immobilize cholesterol oxidase on a conductive polymer to create a cholesterol biosensor. In doing so, they were able to harness the direct electrochemistry of this enzyme to function as sensing material. Co-immobilized microperoxidase was used as a catalyst for the $\mathrm{H}_{2} \mathrm{O}_{2}$ generated by the cholesterol oxidase. The ability to co-immobilize the two enzymes is a promising endeavor for direct electron transfer-based biosensors.

Fan et al. ${ }^{192}$ have demonstrated how $\mathrm{Fe}_{3} \mathrm{O}_{4}-\mathrm{Pt}$ core/shell nanoparticles can be utilized to covalently bind to hemoglobin, retaining the electrochemical behavior of the immobilized protein. The resulting electrode exhibited excellent electrocatalytic activity and stability.

\section{DNA Based Sensors}

DNA biosensors are a relatively new and quickly emerging area due to its simplicity, speed, and economical assays for gene analysis and testing. ${ }^{2}$ DNA biosensors use this high specificity to target sequences in the presence of non-complimentary strands which has a broad range of potential uses from detection of genetic disorders and bacterial and viral infections to forensic and bio-warfare detection. ${ }^{193}$ Recent developments focus on improvement of fabrication and specific biological uses.

To enhance the detection of short DNA sequences Liu et al. ${ }^{194}$ fabricated a DNA biosensor based on hollow gold nanopartices. The hollow gold nanoparticles are fabricated based on a displacement of Co nanoparticle, characterized using CV and electrochemical impedance spectroscopy, have spike-like morphology of their surface which enhances the immobilizations and hybridization of the DNA and after two weeks still maintained about $95 \%$ of their initial response with a wide linear range, $1 \mathrm{pM}$ to $10 \mathrm{nM}$, and low detection limit.

Du et al. ${ }^{195}$ used layer by layer technique to create an electronically conductive and catalytically active polymeric multilayer film by alternating sulfonated polyaniline nanofibers and cysteamine-capped gold nanoparticles, which can electrocatalyze NADH to be used to detect DNA hybridization. This hybridization can be detected using chronopotentiometry, which instantaneously detects DNA hybridization as compared to electrochemical impedance spectroscopy that requires 15-20 minutes before detection.

Current advances in these biosensors have been used to detect DNA for a variety of biological uses with a focus on disease diagnostics some applications are discussed below. Vyskyocil et al. ${ }^{196}$ used a DNA biosensor based on a screen printed CPE immobilized with double-stranded DNA for in detection of genotoxic nitro derivatives. Aladag et al. ${ }^{197}$ used DNA biosensors to detect Apa I polymorphism, which are involved in Alzhiemer's Disease, within 30 minutes, which is more rapid than other current assays. Zhang et al. ${ }^{198}$ used DNA biosensors for simultaneous detections of both HIV-1 and HIV-2 oligonucleotides. Pournaghi-Azar et al. ${ }^{199}$ created a DNA biosensor for direct detection and discrimination of 
hepatitis $\mathrm{C}$ virus genotype $3 \mathrm{a}$. Sun et al. ${ }^{98}$ developed a chitosan/nano- $\mathrm{V}_{2} \mathrm{O}_{5} / \mathrm{MWCNTs}$ composite film modified carbon ionic liquid electrode that could detect Yersinia enterocolitica, a common pathogen in pork known to cause gastroenteritis, with a lower detection limit than current methods. A sensor for the detection of Trichoderma harzianum species which allows for the quantization of microorganisms was developed by Siddiquee et al. 200

\section{Other Papers of Interest}

Yao and $\mathrm{Hu}^{201}$ developed a novel electrode that is $\mathrm{pH}$-switchable, where it is in the on state at $\mathrm{pH} 4.0$ and off at $\mathrm{pH}$ 9.0. They constructed layer by layer films of concanavalin $\mathrm{A}$ and horseradish peroxide on pyrolytic graphene that allows for the control of bioelectrocatalysis of hydrogen peroxide by altering the surrounding $\mathrm{pH}$.

Other interesting developments have involved the use of graphene. Wang et al. ${ }^{109}$ used chemical doping of graphene to modify the intrinsic properties. They used nitrogen plasma treatment of graphene to prepare nitrogen-doped graphene, which displayed high electrocatalytic activity for the reduction of hydrogen peroxide. Also, Wan et al. ${ }^{202}$ used functionalized graphene oxide sheets with a signal amplification method for the sensitive and selective detection of bacteria.

\section{MICROFLUIDIC SYSTEMS}

Microfluidics refers to devices, systems, and methods for the manipulation of fluid, with the length scales in the micrometer range. ${ }^{203}$ Advances in these devices coupled with electrode development has led to the advancement of lab-on-a-chip systems or micrototal analysis systems ( $\mu$ TAS) ${ }^{203,204}$ The use of these systems has found many applications in biochemical analysis, pharmaceutical industry and environmental testing because of their ability to integrate several procedures into a single device. ${ }^{203}$ In the past two years there have been two very thorough reviews on microfluidic systems, one by Liu et al. ${ }^{205}$ in 2010 (145 citations) and the other by Livak-Dahl et al. ${ }^{204}$ in 2011 (149 citations). Livak-Dahl et al. focuses on the overall development of microfluidics while Liu et al. focuses on the recent advances specifically for biosensing and biomedical applications. While there have been a large number of advances in microfluidics in two years, this review will focus on advances in the field that can be coupled with sensors for the development of novel $\mu$ TAS.

\section{Continuous Flow Assays}

Continuous flow microfluidic systems are the main approach for simple biochemical applications, such as diagnostic tests, because they are easy to implement and less sensitive to protein fouling. Unfortunately, they are less suitable for tasks that require a large degree of flexibility. ${ }^{203,205}$ Current advances have focused on creating microfluidic devices that incorporate electrodes into the microfluidic platform to create $\mu \mathrm{TAS}$ for clinical applications.

Lin and Lee ${ }^{206}$ developed a microfluidic device capable of performing online cell counting and continuous cell lysis. To do this, they used a combination of hydrodynamic focusing and an optically induced electric field, where the fibroblast cells were precisely counted and lysed simultaneously in a single chip. They used an applied voltage and illumination power density above $7 \mathrm{~V}$ and $100 \mathrm{~kW} / \mathrm{m}^{2}$ to obtain a lysis rate of $93.8 \%$. Since this device was able to quantify the number of lysed cells, which is essential for the subsequent DNA extraction process, it has potential for various cell-based analysis and molecular diagnosis applications.

Vojtisek et al. ${ }^{207}$ created a rapid microfluidic device for continuous flow DNA hybridization and isolation. They used parallel laminar flow streams containing reagents and buffer 
solutions that were generated in a flow chamber with magnetic particles. These particles tagged with $20 \mathrm{nM}$ single stranded probe DNA were pulled through a stream of complimentary DNA using external magnets while non-specific DNA is washed away in neighboring buffer streams. The bound DNA was fluorescently labeled and detected, which allowed for the detection of DNA down to $20 \mathrm{nmol} / \mathrm{L}$ and greatly reduced total procedural time, compared to conventional methods.

Wang ${ }^{208}$ used a microfluidic device and chelating nanobeads to remove lead from blood. He used a microelectromechanical process to design and fabricate a microfluidic sorter. It has a microchannel with asymmetric electrodes that provided a local dielectrophrosis field strong enough to manipulate the chelating nanobeads and blood cells in a bloodstream flow. The most effective driving velocity for the bloodstream flow was less than $0.09 \mathrm{~m} / \mathrm{s}$ through the device, with an applied power of $5 \mathrm{~W}$ and a frequency of $13.56 \mathrm{MHz}$ ac field to effectively separate out the nanobeads from the bloodstream.

\section{Droplet Assays}

Droplet based microfluidic systems commonly use electrowetting to create picoliter to microliter droplets separated by an immiscible liquid. These droplets remain mobile in the microfluidic system creating isolated chambers without cross contamination or dilution and as a result, offer a unique solution to genomics, proteomics, metabolomics among others. ${ }^{203,204,205}$ Current advances have focused on the use of electrowetting-on-dielectrics (EWOD). EWOD is considered one of the most feasible and efficient methods droplet based assays. EWOD is an actuator based on controlling charge at the interface of liquids and insulators over buried electrodes. EWOD actuators have more flexibility than other common platforms because they can split, mix, and dispense droplets from on-chip reservoirs. A thorough review was published in 2010 by Malic et al. ${ }^{209}$ over these advances in the past decade (126 citations).

Lin et al. ${ }^{210}$ described a method of using low-voltage EWOD with multi-layer insulators to develop devices that could be reused with more reliability. They found that devices which used $135 \mathrm{~nm} \mathrm{Ta} \mathrm{O}_{5}$ and $180 \mathrm{~nm}$ parylene $\mathrm{C}$ as insulators were more robust and operated better under lower applied voltages than device with a homogeneous single dielectric layer. Their devices with $100 \mu \mathrm{m}$ electrode pitch were developed with an actuation threshold voltage of $7.2 \mathrm{~V}$ and a dispensing voltage of $11.4 \mathrm{~V}$, which are lower than the dielectric breakdown voltages of the electrode insulator. They were also able to demonstrate the ability to scale the actuation threshold voltage and physical size of the electrodes to $35 \mu \mathrm{m}$, enabling the transfer of $30 \mathrm{pL}$ droplets.

Karuwan et al. ${ }^{211}$ coupled a three-electrode electrochemical sensing system with EWOD digital microfluidic devices to quantitatively analyze iodide. A sensing system that contained an Au working, Ag reference, and Pt auxiliary wires was suspended at the end of T-junction EWOD mixing device. Microdroplets of Tris buffer and potassium iodide solution were mixed and detected by cyclic voltametry within seconds. Their combination of EWOD digital microfluidic and electrochemical sensing system shows the potential for a novel method of rapid chemical analysis with minimal reagent consumption.

Malk et al. ${ }^{212}$ investigated the characteristic hydrodymamic flow shown in droplets actuated by EWOD with AC voltage. Silicon oil was used as the ambient phase. They found that there was quadripolar flow with four symmetrical vortex structures that was controlled by frequency actuation. Also, they found that droplet oscillations were most likely involved in the hydrodynamic flow. 


\section{Other Papers of Interest}

Liu et al. ${ }^{213}$ developed a microfluidic silicon chip with poly(ethylene glycol) (PEG) hydrogel microarray on the nanoporous anodized aluminum oxide (AAO) membrane for cell based microarray for drug testing. They used photolithography to fabricate PEG hydrogel microstructures on a nanoporous alumina surface modified with a 3-(trimethoxysilyl)propyl methacrylate. Cancer cells were selectively adherent to the 3-(trimethoxysilyl)propyl methacrylate monolayer inside the microwells where they performed diffusion studies using the anti-cancer drug, cisplatin.

Gallaway et al. ${ }^{214}$ designed a single-use microfluidic electrochemical cell to monitor a metal layer electrodeposited from a flowing electrolyte stream, in a lateral configuration, and separated by a flow channel. They found that zinc deposition was effected by flow rate so they used ramified zinc tip approaches, which are independent of electrolyte flow rate. This zinc deposition reaction has a Tafel slope of $130 \mathrm{mV}$ below $10 \mathrm{~mA} / \mathrm{cm}^{2}$ and $50 \mathrm{mV}$ in the second Tafel region less than $10 \mathrm{~mA} / \mathrm{cm}^{2}$ where this second region could have relevance to battery development.

Lee et al. ${ }^{215}$ developed a way of incorporating liquid glass electrodes into fluidic systems. They developed a three-dimensional nanoscale liquid glass electrode by femtosecond-laser nanomachining from monolithic substrates without conductive materials. The electrode consisted of a nanochannel that ended at a nanoscale glass tip, which became a conductor in the presence of high electric fields through dielectric breakdown, and was reversible when the field is removed. They used nanoscale liquid glass electrode to develop a nanoinjector with an electrokinetic pump capable of producing well-controlled flow rates below $1 \mathrm{fL} / \mathrm{s}$. Their electrode could be integrated into other nanodevices and fluidic systems, including actuators and sensors.

\section{MASS SENSORS}

Due to potential uses in the biological and biomedical fields, the development of sensors that can detect mass down to the molecular and atomic level has attracted interest. The overall principle of mass sensors is that the detection of mass changes can be determined by changes in resonance frequency, beam deflection, or electrical resistance from the mass on the surface of the sensor. ${ }^{203,216}$ Two most commonly explored techniques for electrochemical mass sensing are the quartz crystal microbalance (QCM) and cantilevers. QCM utilizes quartz crystal resonators as sensing material while cantilever sensors use piezoresistive cantilevers. ${ }^{203}$ There are other types of mass sensors, however this review will primarily focus on these two.

\section{Quartz Crystal Microbalance}

QCM consists of electrodes plated onto a thin cut disk of quartz. Quartz is a piezoelectric material, meaning that when an oscillating electric field is applied across the surface an acoustic wave is introduced perpendicular to the crystal. If a mass is absorbed or placed onto the surface the change in oscillation frequency is proportional to the amount of mass. ${ }^{203}$ QCM can detect sub-nanogram mass changes and allows for the detection of biomolecules when a receptor protein is immobilized onto the surface. ${ }^{203}$ There have been two review articles in the past two years that thoroughly discuss the developments of QCM, one by Hillman ${ }^{221}$ that discussed the developments in electrochemical QCM (76 citations) and another by Tuantranont et al. ${ }^{217,} 218$ that discussed monolithic multichannel QCM (125 citations). Recent advances have focused on decreasing the limit of detection in assays and improving selectivity with difficult substrates. 
To improve the sensitivity and selectivity for chemical analytes, previous work has looked at various coatings on the QCM surface, including metals, ceramics, and polymers. Wang et al. ${ }^{219}$ looked at electrospun fibrous membranes to improve the sensitivity of a formaldehyde sensor. In particular, they look at polyethyleneimine fibers electrospun with poly(vinyl alcohol), which created a porous surface and was three times more sensitive than the corresponding electrode with a flat surface. The sensor had a range of 10-255 ppm at room temperature and was reversible. The sensor was also selective for formaldehyde over other volatile organic compounds.

Additionally, the selective detection of certain biomarkers in biologically relevant sample posses some challenges. For example, the ability to detect $\mathrm{C}$-reactive proteins in human serum, which are important in inflammation, is difficult due to blood proteins nonspecifically adsorbing onto the surface. Ogi et al. ${ }^{220}$ used an electrodeless QCM sandwich assay with a resonant frequency of $182 \mathrm{MHz}$. Creactive proteins are important biomarkers in inflammation with a threshold level of $30 \mathrm{ng} / \mathrm{mL}$. To combat this, they used a mass-amplified sandwich assay with a biotinated C-reactive protein-antibody whose mass was increased by attaching streptavidin. They were able to detect $\mathrm{C}$-reactive proteins in solution accurately down to $0.1 \mathrm{ng} / \mathrm{mL}$, which is well below the threshold level.

Another difficulty with QCM has to do with the reduced functionality of QCM in a liquid environment. To be able to detect DNA sequences in fluid using QCM, Garcia-Martinez et al. ${ }^{221}$ developed a homemade quartz crystal oscillator circuit where the quartz was completely submerged in liquid. They used Miller oscillator topology and a working frequency of $50 \mathrm{MHz}$ to have a highly sensitive system that detected complimentary DNA concentrations $50 \mathrm{ng} / \mathrm{mL}$ and higher.

Aptamers are synthetic single strand DNA or RNA molecules that fold up into 3-D structures with a high affinity for their target molecules that retain their binding and inhibitory behavior after immobilization making them extremely useful in the development of sensors. Yao et al. ${ }^{222}$ developed a QCM biosensor with aptamers as the recognition element. They compared immunogobulin E detection using an antibody-based sensor and an aptamer-based sensor in human serum. They found that the linear range of the antibody was $10-240 \mu \mathrm{g} / \mathrm{L}$ while it was $2.5-200 \mu \mathrm{g} / \mathrm{L}$ with the aptamer. Also the aptamer based sensor could tolerate repeated regeneration while the antibody based could not.

\section{Cantilever Sensors}

Micro- and nano-cantilever sensors are sensitive mass detectors that have been shown to distinguish mass changes from single cells to single molecules. Cantilever sensors measure the change in mass based on the change in resonant frequency actuated by piezoelectric films. ${ }^{216}$ They are simple, highly sensitive and capable of real time detection. A recent review by Alvarez and Lechuga ${ }^{223}$ was published in 2010 that discussed the overall advances in the field (100 citations). Recent advances have focused on improving the sensing surface and developing better ways to determine mass regardless of position on the cantilever.

A current issue with cantilever sensors is that the mass sensitivity is determined by the location of the mass relative to the free end, creating nonuniform sensitivity. In two current papers, by Parkin and Hahner 228 and Dohn et al., 224,225 they derived general expressions for determining mass regardless of position. Parkin and Hahner ${ }^{224}$ described the development of expressions that allow for the determination of total mass with any mass distributions regardless of the flexural mode, while Dohn et al. ${ }^{225}$ described expressions that allow for the identification of the position of the particles on the cantilever. 
In an article by Joshi et al., ${ }^{226}$ an improvement in the sensing surface of the cantilever for use as bio nano mass sensors by utilizing the mass sensing capabilities of SWCNTs. They looked at the mass sensing capabilities of three different chiral SWCNTs, $(6,4),(7,3)$, and $(8,1)$, to determine which surface yielded the best results at detecting masses on a zeptogram scale. They found that $(8,1)$ SWCNTs yielded a higher frequency shift than the other two chiral SWCNTs.

\section{Other Papers of Interest}

Even though cantilever sensors have been a commonly accepted way of determining mass, there has been a trend in emphasis on ways to replace the cantilever. The main reasons for this eventual shift are the complex calculations required for analysis with cantilevers, along with their nonuniformity and fragility. Schmid et al. ${ }^{227}$ developed a sensor that uses microstrings instead of a cantilever for real-time mass detection of individual particles in air or a vacuum without the need for post-measurement analysis. Silicon nitride micro-strings were successfully used to measure the resonant frequency shifts due to the placement of microparticles on the strings. This method possesses potential uses in the real-time detection of airborne nanoparticles.

In a paper by Melli et al., 228 they describe the use of micro-pillars instead of cantilevers. They used lithography and deep anisotropic etching to fabricate the micro-pillars, which are intrinsically symmetrical, creating uniformity across the surface. Also, they use a selfcalibrating functionalization strategy making them suitable for use in liquid environments, which typically present difficulties in cantilever-based sensors. These micro-pillars have a sensitivity of $33 \mathrm{~Hz} / \mathrm{fg}$ and a reproducibility of $0.1 \mathrm{fg}$ and were selective when tested.

Park et al. ${ }^{229}$ developed micro-electro-mechanical systems, which utilize a four beam-spring sensor structure to retain a uniform mass sensitivity and have the potential to be coupled with microscopy for a comprehensive look at cell growth. They developed this sensor to measure mass, growth rate and biophysical properties of fixed and unfixed cells. The reported sensitivity was $221 \mathrm{~Hz} / \mathrm{ng}$ in liquid. They used this sensor to detect the cell growth of human colorectal carcinoma cells, which increased linearly at a rate of $3.25 \% / \mathrm{hr}$ for over 50 hours.

\section{CONSLUSIONS}

Electrochemical sensors provide a crucial analytical tool as demand for sensitive, rapid, and selective determination of analytes increases. Unlike spectroscopic and chromatographic instruments, electrochemical sensors can be easily adapted for detecting a wide range of analytes, while remaining inexpensive. Additionally, these sensors are capable of being incorporated into robust, portable, or miniaturized devices, enabling tailoring for particular applications.

In the area of potentiometric sensors, emphasis has remained on developing ISEs for the detection of cations, anions, and neutral species. Strategies for incorporating materials to enhance speed, sensitivity, and stability of these sensors has been of particular interest. Additionally, focus on determining the concentration of clinically relevant molecules for drug development, disease screening, and biomedical research has grown in this field. Finally, the further development in E-tongues provides a promising avenue in this field to provide a more general method of detection that can be applied as a more general tool in analytical chemistry.

Previous reviews in this area described the growing importance of exploiting nanomaterials in electrochemical sensors. ${ }^{230,231}$ This trend has both continued and evolved in the past two 
years. As the unique properties of these nanomaterials increases, so does the ability to utilize nanoscience to enhance the properties of electrochemical sensors. Of particular interest are carbon-based materials, which can take numerous morphologies that impact the properties of the resulting sensor. The recent Nobel Prize for graphene has sparked investigations of this new material in a variety of applications, including electrochemical sensors. Moreover, the combination of various nanomaterials into composites in order to explore their synergistic effects has become an interesting area of research.

The incorporation of biomaterials into electrochemical sensors enables the sensitivity and selectivity that are akin to nature. Major advancements in both biosensors and immunosensors revolve around immobilization and interface capabilities of the biological material with the electrode. The use of nanomaterials and sandwich-type devices has provided a means for increasing the signal response from these types of sensors. The ability to incorporate biomaterials with the potential for direct electron transfer is another growing research area in this field.

In general, the field of electrochemical sensors continues to grow and find new areas for application. We believe that the field will focus on the incorporation and interaction of unique materials, both nano and biological, in the coming years. Two branches of electrochemical sensors are developing: sensors with increased specificity and sensors capable of simultaneous/multiplex determination. In both of these branches the ability to operate in complex biological matrixes will remain critical, forcing researchers to solve problems of biocompatibility and stability.

\section{Acknowledgments}

The authors gratefully acknowledge the National Science Foundation (DMR 0907619), the NSF EPSCoR (EPS 1004083), the Research Corporation for Scientific Advancement (Scialog), NIH RC2 DA028981, and the Defense Threat Reduction Acgency (DTRA) HDTRA1-10-0067 for the support of their bioanalytical research.

\section{References}

1. Shan X, Patel U, Wang S, Iglesias R, Tao N. Science. 2010; 327:1363-1366. [PubMed: 20223983]

2. Wang, J. Analytical Electrochemistry. 3. John Wiley \& Sons, Inc; Hoboken: 2006.

3. Bard, AJ.; Faulkner, LR. Electrochemical Methods: Fundamentals and Applications. 2. John Wiley \& Sons, Inc; Hoboken: 2001.

4. Park WJ, Yi Y, Lee J, Lee BC, Park OK, Lee HJ, Lee H. Talanta. 2010; 81:482-485. [PubMed: 20188950]

5. Zhou J, Ren K, Zheng Y, Su J, Ryan D, Wu H. Electrophoresis. 2010; 31:3083-3089. [PubMed: 20803753]

6. Idegami K, Chikae M, Nagatani N, Tamiya E, Takamura Y. Japanese Journal of Applied Physics. 2010; 49:097001-097003.

7. Noh J, Park S, Boo H, Kim HC, Chung TD. Lab Chip. 2010; 11:664-671. [PubMed: 21135953]

8. Vonau W, Oelbner W, Guth U, Henze J. Sensors and Actuators B: Chemical. 2010; 144:368-373.

9. Miura N, Jin H, Wama R, Nakakubo S, Elumalai P, Plashnitsa VV. Sensors and Actuators B: Chemical. 2011; 152:261-266.

10. Rius-Ruiz FX, Kisiel A, Michalska A, Maksymiuk K, Riu J, Rius FX. Anal Bioanal Chem. 2011; 399:3613-3622. [PubMed: 21318254]

11. Muralidharan S, Saraswathy V, Berchmans LJ, Thangavel K, Ann KY. Sensors and Actuators B: Chemical. 2010; 145:225-231.

12. Shibata M, Yamanuki M, Iwamoto Y, Nomura S, Kakiuchi T. Anal Sci. 2010; 26:1203-1206. [PubMed: 21079353]

13. Huang H, Wang Q, Cha CS, Lu J, Zhuang L. Electroanalysis. 2010; 23:577-582. 
14. Pal R, Ananthasivan K, Anthonysamy S, Ganesan V. Electrochim Acta. 2011; 56:4276-4280.

15. Zeng R, Poynton SD, Kizewski JP, Slade RCT, Varcoe JR. Electrochem Commun. 2010; 12:823825 .

16. Bratov A, Abramova N, Ipatov A. Anal Chim Acta. 2010; 678:149-159. [PubMed: 20888446]

17. Faridbod F, Ganjali MR, Pirali-Hamedani M, Norouzi P. Int J Electrochem Sci. 2010; 5:11031112.

18. Norouzi P, Rafiei-Sarmazdeh Z, Faridbod F, Adibi M, Ganjali MR. Int J Electrochem Sci. 2010; 5:367-376.

19. Faridbod F, Ganjali MR, Larijani B, Hosseini M, Norouzi P. Materials Science and Engineering. 2010; 30:555-560.

20. Ganjali MR, Poursaberi T, Khoobi M, Shafiee A, Adibi M, Pirali-Hamedani M, Norouzi P. Int J Electrochem Sci. 2011; 6:717-726.

21. Mashhadizadeh MH, Khani H, Shockravi A. J Incl Phenom Macrocycl Chem. 2010; 68:219-227.

22. Kopylovich MN, Mahmudov KT, Pombeiro AJL. J Hazard Mater. 2011; 186:1154-1162. [PubMed: 21177026]

23. Petkovič BB, Sovilj SP, Budimir MV, Simonovič RM, Jovanovič VM. Electroanalysis. 2010; 22:1894-1900.

24. Hosseini M, Abkenar SD, Ganjali MR, Faridbod F. Materials Science and Engineering. 2011; 31:428-433.

25. Zamani HA, Zabihi MS, Rohani M, Zangeneh-Asadabadi A, Ganjali MR, Faridbod F, Meghdadi S. Materials Science and Engineering. 2011; 31:409-413.

26. Washe AP, Macho S, Crespo GA, Rius FX. Anal Chem. 2010; 82:8106-8112. [PubMed: 20809625]

27. Motlagh MG, Taher MA, Ahmadi A. Electrochim Acta. 2010; 55:6724-6730.

28. Abbaspour A, Mirahmadi E, Khalafi-nejad A, Babamohammadi S. J Hazard Mater. 2010; 174:656-661. [PubMed: 19828247]

29. Ganjali MR, Motakef-Kazami N, Faridbod F, Khoee S, Norouzi P. J Hazard Mater. 2010; 173:415-419. [PubMed: 19758753]

30. Álvarez-Romero GA, Ramirez-Silva MT, Galán-Vidal CA, Páez-Hernández ME, Romero-Romo MA. Electroanalysis. 2010; 22:1650-1654.

31. Zahran EM, Hua Y, Li Y, Flood AH, Bachas LG. Anal Chem. 2010; 82:368-375. [PubMed: 19994863]

32. Kang Y, Lutz C, Hong SA, Sung D, Lee JS, Shin JH, Nam H, Cha GS, Meyerhoff ME. Bull Korean Chem Soc. 2010; 31:1601-1608.

33. Liang RN, Song DA, Zhang RM, Qin W. Angew Chem Int Ed. 2010; 49:2556-2559.

34. Park JY, Azad AM, Song SJ, Wachsman ED. J Am Ceram Soc. 2010; 93:742-749.

35. Madunić-Čačić D, Sak-Bosnar M, Matešić-Pua R. Int J Electrochem Sci. 2011; 6:240-253.

36. Zhuiykov S, Kats E, Marney D. Talanta. 2010; 82:502-507. [PubMed: 20602927]

37. Cunha CO, Silva RCR, Amorim CG, Júnior SA, Araújo AN, Montenegro MCBSM, Silva VL. Electroanalysis. 2010; 22:2967-2972.

38. Kumar KG, Augustine P, John S. J Appl Electrochem. 2010; 40:65-71.

39. Abounassif A, Al-Hadiya BM, Mostafa GAE. Instrum Sci Technol. 2010; 38:165-177.

40. Vlascici D, Pruneanu S, Olenic L, Pogacean F, Ostafe V, Chiriac V, Pica EM, Bolundut LC, Nica L, Fagadar-Cosma E. Sensors. 2010; 10:8850-8864. [PubMed: 22163384]

41. Alizadeh T, Akhoundian M. Electrochim Acta. 2010; 55:3477-3485.

42. Ganjali MR, Faridbod F, Larijani B, Riahi S, Hosseini M, Nasli-Esfahani E, Norouzi P. Int J Electrochem Sci. 2010; 5:200-214.

43. Khaled E, Hassan HNA, Mohamed GG, Ragab FA, Seleim AEA. Talanta. 2010; 83:357-368. [PubMed: 21111146]

44. Wang Y, Chen Q, Zeng X. Biosens Bioelectron. 2010; 25:1356-1362. [PubMed: 19926470]

45. Riul A, Dantas CAR, Miyazaki CM, Oliveira ON. Analyst. 2010; 135:2481-2495. [PubMed: 20730141] 
46. Valle MD. Electroanalysis. 2010; 22:1539-1555.

47. Peres AM, Dias LG, Veloso ACA, Meirinho SG, Morais JS, Machado AASC. Talanta. 2011; 83:857-864. [PubMed: 21147329]

48. Hruškar M, Major N, Krpan M. Talanta. 2010; 81:398-403. [PubMed: 20188937]

49. Polshin E, Rudnitskaya A, Kirsanov D, Legin A, Saison D, Delvaux F, Delvaus FR, Nicolaï BM, Lammertyn J. Talanta. 2010; 81:88-94. [PubMed: 20188892]

50. Gil L, Barat JM, Baigts D, Martínez-Máñez R, Soto J, Garcia-Breijo E, Aristoy M-C, Toldrá F, Llobet E. Food Chem. 2011; 126:1261-1268.

51. Spijkman MJ, Brondijk JJ, Geuns TCT, Smits ECP, Cramer T, Zerbetto F, Stoliar P, Biscarini F, Blom PWM, Leeuw DM. Adv Funct Mater. 2010; 20:898-905.

52. Sakač N, Sak-Bosnar M, Horvat M, Madunić-Čačić D, Szechenyi A, Kovacs B. Talanta. 2011; 83:1606-1612. [PubMed: 21238759]

53. Gupta VK, Jain R, Radhapyari K, Jadon N, Agarwal S. Anal Biochem. 2011; 408:179-196. [PubMed: 20869940]

54. Dogan-Topal B, Ozkan SA, Uslu B. The Open Chemical and Biomedical Methods Journal. 2010; 3:56-73.

55. Alghamdi AH. Arabian Journal of Chemistry. 2010; 3:1-7.

56. Bustos E, Godinez LA. International Journal of Electrochemical Science. 2011; 6:1-36.

57. Jacobs CB, Peairs MJ, Venton BJ. Anal Chim Acta. 2010; 662:105-127. [PubMed: 20171310]

58. Guell AG, Meadows KE, Unwin PR, MacPherson JV. PCCP. 2010; 12:10108-10114. [PubMed: 20689900]

59. Apetrei C, Apetrei IM, Saja JAD, Rodriguez-Mendez ML. Sensors. 2011; 11:1328-1344.

60. Sripirom J, Noor S, Koehler U, Schulte A. Carbon. 2011; 49:2402-2412.

61. Lim CX, Hoh HY, Ang PK, Loh KP. Anal Chem. 2010; 82:7387-7393. [PubMed: 20712323]

62. Ambrosi A, Pumera M. PCCP. 2010; 12:8943-8947. [PubMed: 20532301]

63. Chang J-L, Chang K-H, Hu C-C, Cheng W-L, Zen J-M. Electrochem Commun. 2010; 12:596-599.

64. Wang Y, Wan Y, Zhang D. Electrochem Commun. 2010; 12:187-190.

65. Yue R, Lu Q, Zhou Y. Biosens Bioelectron. 2011; 26:4436-4441. [PubMed: 21612908]

66. Zhang F, Li Y, Gu Y, Wang Z, Wang C. Microchimica Acta. 2011; 173:103-109.

67. Fan Y, Huang K-J, Niu D-J, Yang C-P, Jing Q-S. Electrochim Acta. 2011; 56:4685-4690.

68. Du D, Liu J, Zhang X, Cui X, Lin Y. Journal of Materials Chemistry. 2011; 21:8032-8037.

69. Komathi S, Gopalan AI, Lee K-P. Analyst. 2010; 135:397-404. [PubMed: 20098776]

70. Yang S, Qu L, Li G, Yang R, Liu C. J Electroanal Chem. 2010; 645:115-122.

71. Gong J, Zhou T, Song D, Zhang L, Hu X. Anal Chem. 2010; 82:567-573. [PubMed: 20014816]

72. Huo Z, Zhou Y, Liu Q, He X, Liang Y, Xu M. Microchimica Acta. 2011; 173:119-125.

73. Liu T, Zhu X, Cui L, Ju P, Qu X, Ai S. Journal of Electroanalytical Chemistry. 2011; 651:216221.

74. Guo CX, Lu ZS, Lei Y, Li CM. Electrochem Commun. 2010; 12:1237-1240.

75. Wang Y, Peng W, Liu L, Tang M, Gao F, Li M. Microchimica Acta. 2011; 174:41-46.

76. Olive-Monllau R, Esplandiu MJ, Bartroli J, Baeza M, Cespedes F. Sensors and Actuators, B: Chemical. 2010; B146:353-360.

77. Atta NF, El-Kady MF, Galal A. Anal Biochem. 2010; 400:78-88. [PubMed: 20064483]

78. Noroozifar M, Khorasani-Motlagh M, Taheri A. Talanta. 2010; 80:1657-1664. [PubMed: 20152393]

79. Ulubay S, Dursun Z. Talanta. 2010; 80:1461-1466. [PubMed: 20006114]

80. Kalimuthu P, John SA. Talanta. 2010; 80:1686-1691. [PubMed: 20152397]

81. Rastakhiz N, Kariminik A, Soltani-Nejad V, Roodsaz S. International Journal of Electrochemical Science. 2010; 5:1203-1212.

82. Ghorbani-Bidkorbeh F, Shahrokhian S, Mohammadi A, Dinarvand R. Electrochim Acta. 2010; 55:2752-2759.

83. Ensafi AA, Karimi-Maleh H. J Electroanal Chem. 2010; 640:75-83. 
84. Xie C, Li H, Li S, Wu J, Zhang Z. Anal Chem. 2010; 82:241-249. [PubMed: 19938838]

85. Gomez-Caballero A, Ugarte A, Sanchez-Ortega A, Unceta N, Goicolea MA, Barrio RJ. J Electroanal Chem. 2010; 638:246-253.

86. Hu Y, Li J, Zhang Z, Zhang H, Luo L, Yao S. Anal Chim Acta. 2011; 698:61-68. [PubMed: 21645660]

87. Yang Y, Yi C, Luo J, Liu R, Liu J, Jiang J, Liu X. Biosens Bioelectron. 2011; 26:2607-2612. [PubMed: 21159505]

88. Zhang J, Wang Y, Lv R, Xu L. Electrochim Acta. 2010; 55:4039-4044.

89. Gholivand MB, Torkashvand M. Talanta. 2011; 84:905-912. [PubMed: 21482301]

90. Alizadeh T, Zare M, Ganjali MR, Norouzi P, Tavana B. Biosens Bioelectron. 2010; 25:1166-1172. [PubMed: 19892541]

91. Li J, Jiang F, Li Y, Chen Z. Biosens Bioelectron. 2011; 26:2097-2101. [PubMed: 20932738]

92. Velmurugan J, Zhan D, Mirkin MV. Nat Chem. 2010; 2:498-502. [PubMed: 20489720]

93. Zhang J, Harris AR, Cattrall RW, Bond AM. Analytical Chemistry (Washington, DC, United States). 2010; 82:1624-1633.

94. Mirri G, Bull SD, Horton PN, James TD, Male L, Tucker JHR. J Am Chem Soc. 2010; 132:89038905. [PubMed: 20536177]

95. Pavinatto FJ, Fernandes EGR, Alessio P, Constantino CJL, de SJA, Zucolotto V, Apetrei C, Oliveira ON Jr, Rodriguez-Mendez ML. J Mater Chem. 2011; 21:4995-5003.

96. Alessio P, Pavinatto FJ, Oliveira ON Jr, Saez JADS, Constantino CJL, Rodriguez-Mendez ML. Analyst. 2010; 135:2591-2599. [PubMed: 20714515]

97. Urbanová V, Vytras K, Kuhn A. Electrochem Commun. 2010; 12:114-117.

98. Sun W, Qin P, Gao H, Li G, Jiao K. Biosens Bioelectron. 2010; 25:1264-1270. [PubMed: 19926468]

99. Jin J-H, Kim JH, Park CW, Min NK. Talanta. 2011; 83:1476-1481. [PubMed: 21238739]

100. Amatore C, Arbault Sp, Koh ACW. Anal Chem. 2010; 82:1411-1419. [PubMed: 20102164]

101. Khairy M, Kadara RO, Banks CE. Analytical Methods. 2010; 2:851-854.

102. Colombo E, Men Y, Scharpf J, Pietzka C, Dipalo M, Herfurth P, Gao Z, Schneider M, Carabelli V, Carbone E, Kohn E, Pasquarelli A. Diamond Relat Mater. 20:793-797.

103. Windmiller JR, Zhou N, Chuang M-C, Valdes-Ramirez G, Santhosh P, Miller PR, Narayan R, Wang J. Analyst. 2011; 136:1846-1851. [PubMed: 21412519]

104. Habibi B, Pezhhan H, Pournaghi-Azar M. Microchimica Acta. 2010; 169:313-320.

105. Guo Y, Guo S, Fang Y, Dong S. Electrochim Acta. 2010; 55:3927-3931.

106. Tang X, Liu Y, Hou H, You T. Talanta. 2010; 80:2182-2186. [PubMed: 20152470]

107. Liu Y, Wang D, Huang J, Hou H, You T. Electrochem Commun. 2010; 12:1108-1111.

108. Brownson DAC, Banks CE. Analyst. 2011; 136:2084-2089. [PubMed: 21461417]

109. Tang Y, Pan K, Wang X, Liu C, Luo S. Microchimica Acta. 2010; 168:231-237.

110. Tao B, Zhang J, Hui S, Chen X, Wan L. Electrochim Acta. 2010; 55:5019-5023.

111. Sattarahmady N, Heli H, Moosavi-Movahedi AA. Biosens Bioelectron. 2010; 25:2329-2335. [PubMed: 20413292]

112. Toghill KE, Compton RG. International Journal of Electrochemical Science. 2010; 5:1246-1301.

113. Yang J, Zhang W-D, Gunasekaran S. Biosens Bioelectron. 2010; 26:279-284. [PubMed: 20615684]

114. Wang G, Wei Y, Zhang W, Zhang X, Fang B, Wang L. Microchimica Acta. 2010; 168:87-92.

115. Jiang L-C, Zhang W-D. Biosens Bioelectron. 2010; 25:1402-1407. [PubMed: 19942424]

116. El Khatib KM, Abdel Hameed RM. Biosens Bioelectron. 2011; 26:3542-3548. [PubMed: 21353522]

117. Xu L, Xia J, Li H, Li H, Wang K, Yin S. Eur J Inorg Chem. 2011; 2011:1361-1365.

118. Chen, X-m; Lin, Z-j; Chen, D-J.; Jia, T-t; Cai, Z-m; Wang, X-r; Chen, X.; Chen, G-n; Oyama, M. Biosens Bioelectron. 2010; 25:1803-1808. [PubMed: 20080042]

119. Yang J, Zhang W-d, Gunasekaran S. Electrochim Acta. 2011; 56:5538-5544. 
120. Wang X, Zhang Y, Banks CE, Chen Q, Ji X. Colloids and Surfaces B: Biointerfaces. 2010; 78:363-366.

121. Mu Y, Jia D, He Y, Miao Y, Wu H-L. Biosens Bioelectron. 2011; 26:2948-2952. [PubMed: 21167705]

122. Seo B, Kim J. Electroanalysis. 2010; 22:939-945.

123. Meng F, Yan X, Liu J, Gu J, Zou Z. Electrochim Acta. 2011; 56:4657-4662.

124. Lin C-Y, Lai Y-H, Balamurugan A, Vittal R, Lin C-W, Ho K-C. Talanta. 2010; 82:340-347. [PubMed: 20685476]

125. Bo X, Bai J, Wang L, Guo L. Talanta. 2010; 81:339-345. [PubMed: 20188929]

126. Salimi A, Mahdioun M, Noorbakhsh A, Abdolmaleki A, Ghavami R. Electrochim Acta. 2011; 56:3387-3394.

127. Xu B, Ye M-L, Yu Y-X, Zhang W-D. Anal Chim Acta. 2010; 674:20-26. [PubMed: 20638494]

128. Cui L, Yin H, Dong J, Fan H, Liu T, Ju P, Ai S. Biosens Bioelectron. 2011; 26:3278-3283. [PubMed: 21247748]

129. Salazar P, Martín M, Roche R, O’Neill RD, González-Mora JL. Electrochim Acta. 2010; 55:6476-6484.

130. Jiang Y, Zhang X, Shan C, Hua S, Zhang Q, Bai X, Dan L, Niu L. Talanta. 2011; 85:76-81. [PubMed: 21645672]

131. Hartwell SK, Grudpan K. Microchim Acta. 2010; 169:201-220.

132. Ji SR, Liu C, Zhang B, Yang F, Xu J, Long J, Jin C, Fu DL, Ni QX, Yu XJ. Biochim Biophys Acta. 2010; 1806:29-35. [PubMed: 20193746]

133. Serafín V, Eguílaz M, Agüí L, Yáñez-Sedeño P, Pingarrón JM. Electroanalysis. 2010; 23:169_ 176.

134. Jie G, Liu P, Wang L, Zhang S. Electrochem Commun. 2010; 12:22-26.

135. Malhotra R, Patel V, Vaqué JP, Gutkind JS, Rusling JF. Anal Chem. 2010; 82:3118-3123. [PubMed: 20192182]

136. Che X, Yuan R, Chai Y, Li J, Song Z, Wang J. J Colloid Interface Sci. 2010; 345:174-180.

137. Giannetto M, Elviri L, Careri M, Mangia A, Mori G. Biosens Bioelectron. 2011; 26:2232-2236. [PubMed: 20947323]

138. Du D, Zou Z, Shin Y, Wang J, Wu H, Engelhard MH, Liu J, Aksay IA, Lin Y. Anal Chem. 2010; 82:2989-2995. [PubMed: 20201502]

139. Tang J, Su B, Tang D, Chen G. Biosens Bioelectron. 2010; 25:2657-2662. [PubMed: 20483583]

140. Wei Q, Mao K, Wu D, Dai Y, Yang J, Du B, Yang M, Li H. Sensors and Actuators B: Chemical. 2010; 149:314-318.

141. Laboria N, Fragoso A, Kemmner W, Latta D, Nilsson O, Luz Botero M, Drese K, O'Sullivan CK. Anal Chem. 2010; 82:1712-1719. [PubMed: 20112971]

142. Liao Y, Yuan R, Chai Y, Zhuo Y, Yang X. Anal Biochem. 2010; 402:47-53. [PubMed: 20230773]

143. Song Z, Yuan R, Chai Y, Yin B, Fu P, Wang J. Electrochim Acta. 2010; 55:1778-1784.

144. Zhong Z, Wu W, Wang D, Wang D, Shan J, Zhang Z. Biosens Bioelectron. 2010; 25:2379-2383. [PubMed: 20353889]

145. Li Q, Zeng L, Wang J, Tang D, Liu B, Chen G, Wei M. ACS Applied Materials \& Interfaces. 2011; 3:1366-1373. [PubMed: 21446771]

146. Gu B, Xu C, Yang C, Liu S, Wang M. Biosens Bioelectron. 2011; 26:2720-2723. [PubMed: 20961745]

147. Yang M, Javadi A, Gong S. Sensors and Actuators B: Chemical. 2011; 155:357-360.

148. Yin Z, Liu Y, Jiang LP, Zhu JJ. Biosens Bioelectron. 2011; 26:1890-1894. [PubMed: 20378330]

149. Tang D, Tang J, Su B, Chen G. J Agric Food Chem. 2010; 58:10824-10830.

150. Ahirwal GK, Mitra CK. Biosens Bioelectron. 2010; 25:2016-2020. [PubMed: 20171869]

151. Wang L, Jia X, Zhou Y, Xie Q, Yao S. Microchimica Acta. 2010; 168:245-251.

152. Yang M, Li H, Javadi A, Gong S. Biomaterials. 2010; 31:3281-3286. [PubMed: 20116845]

Anal Chem. Author manuscript; available in PMC 2013 January 17. 
153. Su B, Tang D, Li Q, Tang J, Chen G. Anal Chim Acta. 2011; 692:116-124. [PubMed: 21501720]

154. Huang J, Lin Q, Zhang X, He X, Xing X, Lian W, Zuo M, Zhang Q. Food Research International. 2011; 44:92-97.

155. Rezaei B, Majidi N, Rahmani H, Khayamian T. Biosens Bioelectron. 2011; 26:2130-2134. [PubMed: 20933383]

156. Loyprasert S, Hedström M, Thavarungkul P, Kanatharana P, Mattiasson B. Biosens Bioelectron. 2010; 25:1977-1983. [PubMed: 20167466]

157. Wan Y, Lin Z, Zhang D, Wang Y, Hou B. Biosens Bioelectron. 2011; 26:1959-1964. [PubMed: 20888216]

158. Shao Y, Wang J, Wu H, Liu J, Aksay IA, Lin Y. Electroanalysis. 2010; 22:1027-1036.

159. Zhao Z, Lei W, Zhang X, Wang B, Jiang H. Sensors. 2010; 10:1216-1231. [PubMed: 22205864]

160. Siqueira JR, Caseli L, Crespilho FN, Zucolotto V, Oliveira ON. Biosens Bioelectron. 2010; 25:1254-1263. [PubMed: 19889526]

161. Harper A, Anderson MR. Sensors. 2010; 10:8248-8274. [PubMed: 22163652]

162. Singh RP, Oh BK, Choi JW. Bioelectrochemistry. 2010; 79:153-161. [PubMed: 20356802]

163. Park BW, Yoon DY, Kim DS. Biosens Bioelectron. 2010; 26:1-10. [PubMed: 20554194]

164. Su L, Jia W, Hou C, Lei Y. Biosens Bioelectron. 2011; 26:1788-1799. [PubMed: 20951023]

165. Li Z, Wang X, Wen G, Shuang S, Dong C, Paau MC, Choi MMF. Biosens Bioelectron. 2011; 26:4619-4623. [PubMed: 21612909]

166. Guo CX, Sheng ZM, Shen YQ, Dong ZL, Li CM. ACS Applied Materials \& Interfaces. 2010; 2:2481-2484. [PubMed: 20722406]

167. Ahmad M, Pan C, Luo Z, Zhu J. The Journal of Physical Chemistry C. 2010; 114:9308-9313.

168. Baby TT, Ramaprabhu S. Talanta. 2010; 80:2016-2022. [PubMed: 20152447]

169. Zou C, Fu Y, Xie Q, Yao S. Biosens Bioelectron. 2010; 25:1277-1282. [PubMed: 19926469]

170. Tang H, Yan F, Tai Q, Chan HLW. Biosens Bioelectron. 2010; 25:1646-1651. [PubMed: 20045308]

171. Wang Y, Yuan R, Chaia Y, Li W, Zhuo Y, Yuan Y, Li J. J Mol Catal B: Enzym. 2010; 71:146151.

172. Wan D, Yuan S, Li GL, Neoh KG, Kang ET. ACS Applied Materials \& Interfaces. 2010; 2:30833091. [PubMed: 20964413]

173. Su S, He Y, Song S, Li D, Wang L, Fan C, Lee S-T. Nanoscale. 2010; 2:1704-1707. [PubMed: 20689869]

174. Murphy-Perez E, Arya SK, Bhansali S. Analyst. 2011; 136:1686-1689. [PubMed: 21369619]

175. Zhang ZB, Yuan SJ, Zhu XL, Neoh KG, Kang ET. Biosens Bioelectron. 2010; 25:1102-1108. [PubMed: 19837578]

176. Romero MR, Ahumada F, Garay F, Baruzzi AM. Anal Chem (Washington, DC, U S). 2010; 82:5568-5572.

177. Gamero M, Pariente F, Lorenzo E, Alonso C. Biosens Bioelectron. 2010; 25:2038-2044. [PubMed: 20171079]

178. Yashina EI, Borisova AV, Karyakina EE, Shchegolikhina OI, Vagin MY, Sakharov DA, Tonevitsky AG, Karyakin AA. Anal Chem (Washington, DC, U S). 2010; 82:1601-1604.

179. Haghighi B, Bozorgzadeh S. Talanta. 2011; 85:2189-2193. [PubMed: 21872077]

180. Sheng Q-L, Zheng J-B, Shang-Guan X-D, Lin W-H, Li Y-Y, Liu R-X. Electrochim Acta. 2010; 55:3185-3191.

181. Safavi A, Farjami F. Anal Biochem. 2010; 402:20-25. [PubMed: 20230770]

182. Akyilmaz E, Yorganci E, Asav E. Bioelectrochemistry. 2010; 78:155-160. [PubMed: 19840905]

183. Akyilmaz E, Kozgus O, Turkmen H, Cetinkaya B. Bioelectrochemistry. 2010; 78:135-140. [PubMed: 19783226]

184. Di Fusco M, Tortolini C, Deriu D, Mazzei F. Talanta. 2010; 81:235-240. [PubMed: 20188914]

185. Lu L, Zhang L, Zhang X, Wu Z, Huan S, Shen G, Yu R. Electroanalysis. 2010; 22:471-477. 
186. Kafi AKM, Ahmadalinezhad A, Wang J, Thomas DF, Chen A. Biosens Bioelectron. 2010; 25:2458-2463. [PubMed: 20435460]

187. Ding Y, Wang Y, Li B, Lei Y. Biosens Bioelectron. 2010; 25:2009-2015. [PubMed: 20167467]

188. Ndangili PM, Waryo TT, Muchindu M, Baker PGL, Ngila CJ, Iwuoha EI. Electrochim Acta. 2010; 55:4267-4273.

189. Won Y-H, Aboagye D, Jang HS, Jitianu A, Stanciu LA. J Mater Chem. 2010; 20:5030-5034.

190. Silveira CM, Gomes SP, Araújo AN, Montenegro MCBSM, Todorovic S, Viana AS, Silva RJC, Moura JJG, Almeida MG. Biosens Bioelectron. 2010; 25:2026-2032. [PubMed: 20188536]

191. Abdelwahab AA, Won M-S, Shim Y-B. Electroanalysis. 2010; 22:21-25.

192. Fan H, Pan Z-Q, Gu H-Y. Microchimica Acta. 2010; 168:239-244.

193. Abu Salah K, Alrokyan SA, Khan MN, Ansari AA. Sensors. 2010; 10:963-993.

194. Liu S, Liu J, Han X, Cui Y, Wang W. Biosens Bioelectron. 2010; 25:1640-1645. [PubMed: 20034780]

195. Du M, Yang T, Jiao K. Talanta. 2010; 81:1022-1027. [PubMed: 20298888]

196. Vyskocil V, Labuda J, Barek J. Anal Bioanal Chem. 2010; 397:233-241. [PubMed: 20186538]

197. Aladag N, Ozkan-Ariksoysal D, Gezen-Ak D, Yilmazer S, Ozsoz M. Electroanalysis. 2010; 22:590-598.

198. Zhang D, Peng Y, Qi H, Gao Q, Zhang C. Biosens Bioelectron. 2010; 25:1088-1094. [PubMed: 19850463]

199. Pournaghi-Azar MH, Ahour F, Hejazi MS. Anal Bioanal Chem. 2010; 397:3581-3587. [PubMed: 20563795]

200. Siddiquee S, Yusof NA, Salleh AB, Abu BF, Heng LY. Bioelectrochemistry. 2010; 79:31-36. [PubMed: 19945357]

201. Yao H, Hu N. The Journal of Physical Chemistry B. 2010; 114:3380-3386. [PubMed: 20163095]

202. Wan Y, Wang Y, Wu J, Zhang D. Anal Chem. 2010; 83:648-653. [PubMed: 21175166]

203. Marks, RS.; Cullen, DC.; Karube, I.; Lowe, CR.; Weetall, HH. Handbook of Biosensors and Biochips. John Wiley and Sons; London, UK: 2007.

204. Livak-Dahl E, Sinn I, Burns M. Annu Rev Chem Biomol Eng. 2011; 2:325-353.

205. Liu K-K, Wu R-G, Chuang Y-J, Khoo HS, Huang S-H, Tseng F-G. Sensors. 2010; 10:66236661. [PubMed: 22163570]

206. Lin YH, Lee GB. Sensors and Actuators B-Chemical. 2010; 145:854-860.

207. Vojtisek M, Iles A, Pamme N. Biosens Bioelectron. 2010; 25:2172-2176. [PubMed: 20181469]

208. Wang MW. J Electrochem Soc. 2011; 158:D166-D171.

209. Malic L, Brassard D, Veres T, Tabrizian M. Lab on a Chip. 2010; 10:418-431. [PubMed: 20126681]

210. Lin Y-Y, Evans RD, Welch E, Hsu B-N, Madison AC, Fair RB. Sensors and Actuators B: Chemical. 2010; 150:465-470.

211. Karuwan C, Sukthang K, Wisitsoraat A, Phokharatkul D, Patthanasettakul V, Wechsatol W, Tuantranont A. Talanta. 2011; 84:1384-1389. [PubMed: 21641456]

212. Malk R, Fouillet Y, Davoust L. Sensors and Actuators B-Chemical. 2011; 154:191-198.

213. Liu ZB, Zhang Y, Yu JJ, Mak AFT, Li Y, Yang M. Sensors and Actuators B-Chemical. 2010; 143:776-783.

214. Gallaway JW, Desai D, Gaikwad A, Corredor C, Banerjee S, Steingart D. J Electrochem Soc. 2010; 157:A1279-A1286.

215. Lee S, An R, Hunt AJ. Nature Nanotechnology. 2010; 5:412-416.

216. Hahm, J-i. Sensors. 2011; 11:3327-3355. [PubMed: 21691441]

217. Hillman AR. J Solid State Electrochem. 2011; 15:1647-1660.

218. Tuantranont A, Wisitsora-at A, Sritongkham P, Jaruwongrungsee K. Anal Chim Acta. 2011; 687:114-128. [PubMed: 21277413]

219. Wang XF, Ding B, Sun M, Yu JY, Sun G. Sensors and Actuators B-Chemical. 2010; 144:11-17. 
220. Ogi H, Yanagida T, Hirao M, Nishiyama M. Biosens Bioelectron. 2011; 26:4819-4822. [PubMed: 21680171]

221. Garcia-Martinez G, Bustabad EA, Perrot H, Gabrielli C, Bucur B, Lazerges M, Rose D, Rodriguez-Pardo L, Fariña J, Compère C, Vives AA. Sensors. 2011; 11:7656-7664. [PubMed: 22164037]

222. Yao C, Zhu T, Qi Y, Zhao Y, Xia H, Fu W. Sensors. 2010; 10:5859-5871. [PubMed: 22219691]

223. Alvarez M, Lechuga LM. Analyst. 2010; 135:827-836. [PubMed: 20419229]

224. Parkin JD, Haehner G. Rev Sci Instrum. 2011; 82:035108/035101-035108/035104. [PubMed: 21456790]

225. Dohn S, Schmid S, Amiot F, Boisen A. Appl Phys Lett. 2010; 97:044103/044101044103/044103.

226. Joshi AY, Sharma SC, Harsha SP. Sens Actuators, A. 2011; A168:275-280.

227. Schmid S, Dohn S, Boisen A. Sensors. 2010; 10:8092-8100. [PubMed: 22163642]

228. Melli M, Pozzato A, Lazzarino M. Microelectron Eng. 2010; 87:730-733.

229. Park K, Millet LJ, Kim N, Li H, Jin X, Popescu G, Alurud NR, Hsia KJ, Bashir R. Proc Natl Acad Sci U S A. 2010; 107:20691-20696. S20691/20691-S20691/20612. [PubMed: 21068372]

230. Privett BJ, Shin JH, Schoenfisch MH. Anal Chem. 2008; 80:4499-4517. [PubMed: 18491869]

231. Privett BJ, Shin JH, Schoenfisch MH. Anal Chem. 2010; 82:4723-4741. [PubMed: 20476724]

\section{Biographies}

Danielle W. Kimmel received her BS in chemistry with a concentration in biochemistry at the University of Louisville in 2007. Under the guidance of Dr. Brian Bachmann she earned her MS in chemistry from Vanderbilt University in 2009 studying secondary metabolites produced by bacterial species. Currently, she is a Ph.D. candidate in the Department of Chemistry at Vanderbilt University under the guidance of Dr. David Cliffel. Her research focuses on using multianalyte microphysiometry instrumentation to study dynamic metabolism of macrophages undergoing oxidative stress.

Gabriel LeBlanc received his B.S. in chemistry at Lyon College in 2010. Currently, he is a graduate student in the Department of Chemistry at Vanderbilt University under the direction of Dr. David Cliffel. His research focuses on the integration of photoactive membrane proteins with various electrode materials for solar energy conversion.

Mika Meschievitz received a B.S. in chemistry from Middle Tennessee State University in 2009. Currently she is a graduate student in the Department of Chemistry at Vanderbilt University under the guidance of Dr. David Cliffel. Her current research focuses on using multianalyte microphysiometry to study the metabolic effects of bacteria-host interactions.

David Cliffel is an Associate Professor of Chemistry at Vanderbilt University. He received a BS in Chemistry and a Bachelor of Electrical Engineering from the University of Dayton in 1992. He received his Ph.D. in chemistry in 1998 from the University of Texas at Austin under the direction of Professor Allen J. Bard, and did postdoctoral work with Professor Royce W. Murray at the University of North Carolina as a postdoctoral associate working on the electrochemistry of monolayer protected clusters. His current research concentrates on the electrochemical analysis of nanoparticles and of biological cells using scanning electrochemical microscopy, and has developed the multianalyte microphysiometer for metabolic profiling and toxicology. 


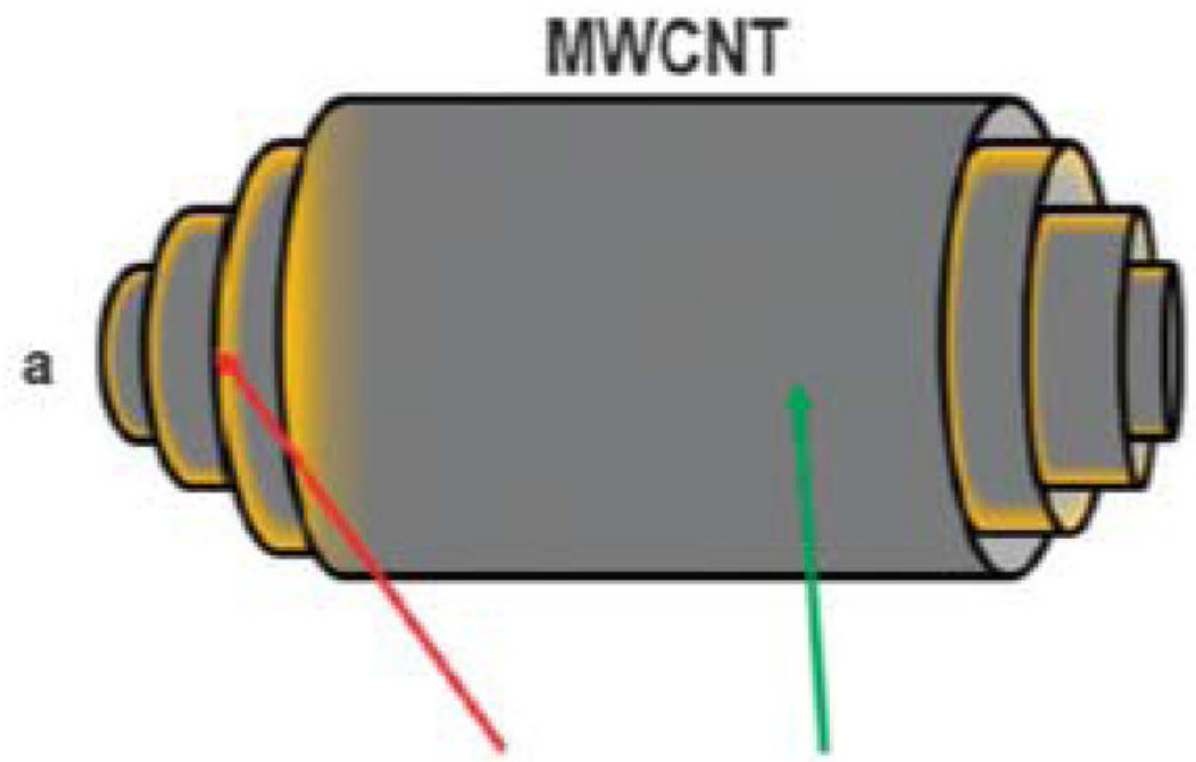

\section{Edge Basal plane}

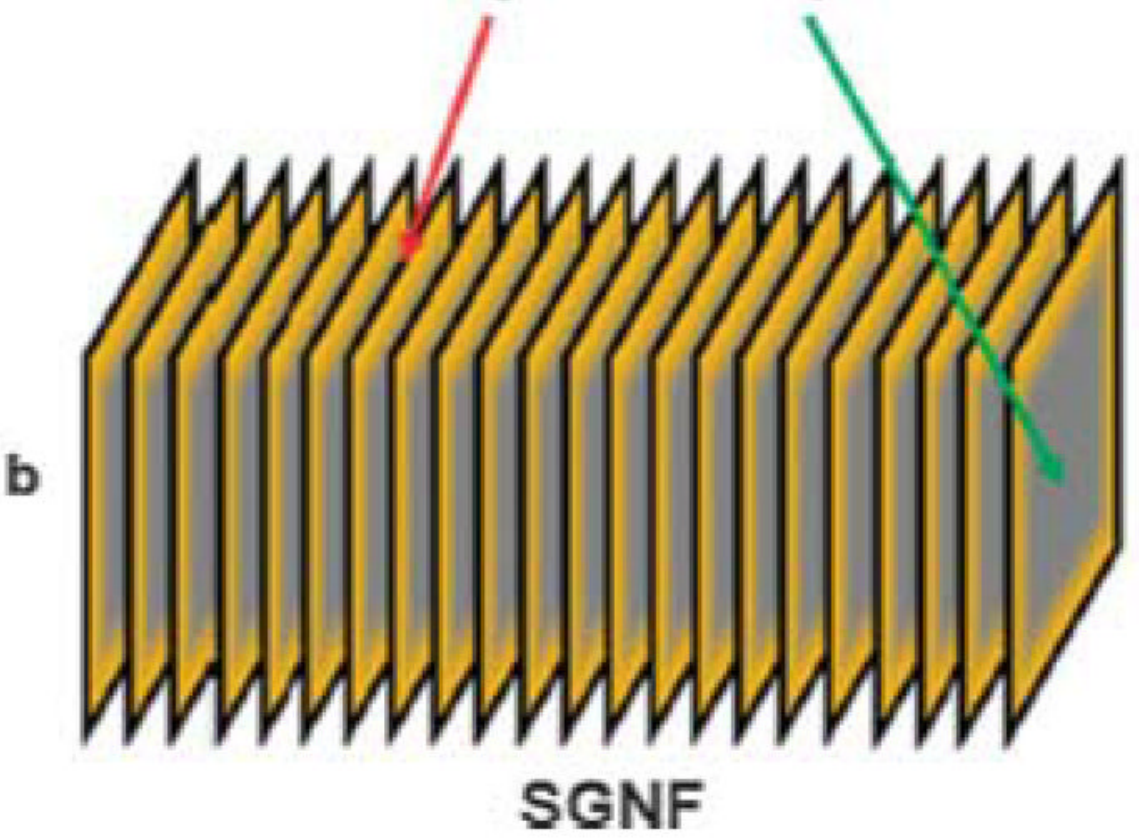

Figure 1.

Schematics of graphene sheet orientation in MWCNTs (a) and stacked graphene nanofibers (SGNF), b). The highly electroactive edge portion of the sheets are highlighted in yellow. Reproduced by permission of the PCCP Owner Societies. ${ }^{62}$ http://dx.doi.org/10.1039/C0CP00213E 
Table 1

List of abbreviations used in this review

\begin{tabular}{|l|l|}
\hline Abbreviation & Meaning \\
\hline ChEs & cholinesterases \\
\hline CNT & carbon nanotube \\
\hline CPE & carbon paste electrode \\
\hline EDTA & ethylenediaminetetraacetic acid \\
\hline E-tongues & electronic tongues \\
\hline EWOD & electrowetting-on-dielectric \\
\hline GC & glassy carbon \\
\hline GOx & glucose oxidase \\
\hline IgG & Immunoglobulin G \\
\hline ISE & ion selective electrode \\
\hline ITO & indium tin oxide \\
\hline LB & Langmuir Blodgett \\
\hline LDH & layered doubled hydroxides \\
\hline Lox & lactate oxidase \\
\hline MIP & molecularly imprinted polymers \\
\hline MWCNT & multi-walled carbon nanotube \\
\hline NADH & nicotinamide adenine dinucleotide \\
\hline pBDD & polycrystalline boron doped diamond \\
\hline PPT & 1-phenyl-3-pyridin-2-yl-thiourea \\
\hline PVC & polyvinyl chloride \\
\hline QCM & quartz crystal microbalance \\
\hline RTIL & room temperature ionic liquids \\
\hline SiNWs & silicon nanowires \\
\hline SPR & surface plasmon resonance \\
\hline SSRE & solid-state reference electrode \\
\hline SWCNT & single-walled carbon nanotube \\
\hline TNF- $\alpha$ & tumor necrosis factor- $\alpha$ \\
\hline TNT & ultramicroelectrodes \\
\hline UMEs & \\
\hline$\mu$ TAS & mitrotoluene \\
\hline analysis systems \\
\hline SW
\end{tabular}

